\title{
Article \\ Application of Multilayer Evidence for Annotation of C-Terminal BRCA2 Variants
}

\author{
Henriett Butz ${ }^{1,2,3,+} \mathbb{D}$, János Papp ${ }^{1,2,+}$, Anikó Bozsik ${ }^{1,2} \mathbb{D}$, Lilla Krokker ${ }^{2,3}$, Tímea Pócza ${ }^{1}$, Edit Oláh ${ }^{1}$ and \\ Attila Patócs 1,2,3,*
}

1 Department of Molecular Genetics, National Institute of Oncology, H-1122 Budapest, Hungary; butz.henriett@med.semmelweis-univ.hu (H.B.); janos.papp@oncol.hu (J.P.); bozsik.aniko@oncol.hu (A.B.); timi.pocza@gmail.com (T.P.); e.olah@oncol.hu (E.O.)

2 Hereditary Cancers Research Group, Hungarian Academy of Sciences-Semmelweis University, H-1089 Budapest, Hungary; krkkr.lilla@gmail.com

3 Department of Laboratory Medicine, Semmelweis University, H-1089 Budapest, Hungary

* Correspondence: patocs.attila@med.semmelweis-univ.hu

+ These authors contributed equally to this work.

check for updates

Citation: Butz, H.; Papp, J.; Bozsik, A.; Krokker, L.; Pócza, T.; Oláh, E.; Patócs, A. Application of Multilayer Evidence for Annotation of C-Terminal BRCA2 Variants. Cancers 2021, 13, 881. https://doi.org/ $10.3390 /$ cancers 13040881

Academic Editor: David Wong

Received: 31 December 2020

Accepted: 15 February 2021

Published: 20 February 2021

Publisher's Note: MDPI stays neutra with regard to jurisdictional claims in published maps and institutional affiliations.

Copyright: (C) 2021 by the authors Licensee MDPI, Basel, Switzerland. This article is an open access article distributed under the terms and conditions of the Creative Commons Attribution (CC BY) license (https:// creativecommons.org/licenses/by/ $4.0 /)$.
Simple Summary: The potential pathogenic role of germline BRCA2 c.9976A $>$ T and c.10095delins GAATTATATCT was evaluated in hereditary breast and ovarian cancer (HBOC) patients by investigating 2491 probands and verified in an independent cohort of 122,209 patients. Although the c.10095delinsGAATTATATCT variant was more prevalent among patients compared to control populations, no increased risk for cancer was found. No association between c.9976A $>$ T and clinicopathological parameters or elevated risk for HBOC cases was detected. However, lung cancer was more prevalent in families carrying c.9976A $>$ T compared to pathogenic BRCA1/BRCA2 carrier families. An increased frequency of pancreatic cancer was found in families where c.9976A $>$ T occurred together with other pathogenic BRCA1 variants. The C-terminal stop codon variants showed no association with other pathogenic BRCA2 variants. No loss of heterozygosity $(\mathrm{LOH})$ in tumor tissue and no allelic imbalance in RNA level were confirmed. The c.9976A $>$ T variant may be considered as a potential risk for lung cancer, and a potential modifying factor in pancreatic cancer when it occurs along with the pathogenic $B R C A 1$ variant, although this observation should be validated in a larger sample cohort.

Abstract: The clinical relevance of the BRCA2 C-terminal stop codon variants is controversial. The pathogenic role of the germline BRCA2 c.9976A $>$ T and c.10095delinsGAATTATATCT variants in hereditary breast and ovarian cancer (HBOC) patients was evaluated. An association with clinicopathological parameters was performed in 2491 independent probands diagnosed with HBOC and in 122,209 cancer patients reported earlier. Loss-of-heterozygosity (LOH) in tumor samples and allelic imbalance in RNA extracted from peripheral blood cells were investigated. Neither c.10095delinsGAATTATATCT or c.9976A > T variants showed significant association with clinicopathological parameters or elevated risk for HBOC-associated tumors. Lung cancer was more prevalent in families carrying the c.9976A $>$ T variant compared to pathogenic BRCA1 or BRCA2 carrier families. An increased prevalence of pancreatic cancer was found in families where c.9976A $>\mathrm{T}$ occurred together with other pathogenic BRCA1 variants. An increased risk for familial pancreatic, lung and upper aero-digestive tract cancers was confirmed in the validation set. Regarding $B R C A 2$ $C$-terminal variants, no linkage with other pathogenic $B R C A 2$ variants, no $\mathrm{LOH}$ in tumor tissue and no allelic imbalance in RNA level were confirmed. The c.9976 A $>$ T variant may be considered as a potential risk for lung cancer, and a potential modifying factor in pancreatic cancer when it occurs along with the pathogenic $B R C A 1$ variant, although this observation should be validated in a larger sample cohort.

Keywords: BRCA2; breast cancer; ovarian cancer; p.K3326*; cancer predisposition; NGS 


\section{Introduction}

In the American College of Medical Genetics and Genomics (ACMG) classification system stop codon (truncation) variants are usually considered to be pathogenic/likely pathogenic [1]. Additionally, stop codon variants of the $B R C A 2$ gene are frequent among all pathogenic variants, leading to a significant increase in the risk of breast and ovarian cancer. However, damaging variants of the C-terminal of the BRCA2 gene have not been investigated or are not considered pathogenic due to Evidence-based Network for the Interpretation of Germline Mutant Alleles (ENIGMA) classification [2]. Among their criteria, they suggest that "a variant predicted to disrupt expression only of protein sequence downstream of position 3325 would be considered unlikely to be clinically important. Further functional and clinical studies are underway to refine risk, if any, for predicted nonsense or frameshift variants downstream of position 3326". The BRCA2 protein has multiple roles besides the well-known DNA double-stranded break repair by homologous recombination, such as maintaining genome stability, including DNA replication, telomere homeostasis and cell cycle progression [3]. These functions have been investigated by different assays but not all functions are available for exploration due to either technical or study limitations. The C-terminal of the BRCA2 protein contains interaction sites of RAD51 and multiple phosphorylation sites affecting their function [4-6]. While, among these, S3291 probably has the highest impact in the BRCA2-RAD51 interaction, a protein sequence of amino acids 3265-3330 of the BRCA2 protein was also reported to bind RAD51 [4-6]. Additionally, a serine at the position of 3397 located more terminally from c.9976A>T $\left(\mathrm{K} 3326^{*}\right)$ is also a phosphorylation site, the function of which has been poorly investigated (https:/ / www.phosphosite.org (accessed on 30 December 2020)). Furthermore, the interaction of the BRCA2 C-terminal with RAD51 may be less significant for homology-directed repair (HDR) than for the protection of stalled replication forks, a relatively newly discovered and HDR-independent function of BRCA2 [7]. Stalled replication fork degradation occurs due to MRE11 nuclease in the lack of $B R C A 2$-mediated fork protection, in which its C-terminus has an essential role [7]. Although the defect of this function of $B R C A 2$ did not lead to cell survival change, the frequency of chromosomal aberrations was found to be increased [7]. It was suggested that $B R C A 2$ protein defective in maintaining fork stability and still proficient in HDR would be insensitive to Poly (ADP-ribose) polymerase (PARP) inhibitors, which specifically exploit the defect of double-strand repair [7].

Previous literature data regarding the clinical relevance of $B R C A 2$ c.9976A $>$ T Cterminal stop codon variants have remained controversial, suggesting either a potential pathogenic role [8-10] or no clinical significance [11-13]. The BRCA2 c.9976A $>$ T variant results in a stop codon at amino acid position 3326. Initially, it was considered pathogenic due to its nonsense coding nature, however, it was reclassified as non-pathogenic based on case-control studies [11]. Among previously published literature, breast cancer risk was elevated for BRCA2 c.9976A $>$ T carriers when compared to a control population in three reports $[9,14,15]$. Studies investigating only ovarian cancer patients, except of Stafford et al. (2017) [16], showed similar odds ratios (ORs). Interestingly, among familial pancreatic, lung and upper aero-digestive tract (UADT) cancer patients, c.9976A $>$ T carrier status was associated with increased risk for developing cancer [8,10,17].

Using basic classification rules, the c.10095delins GAATTATATCT variant, due to its nature (a combination of a deletion and an insertion leading to frame shift and consequently a premature stop codon, Figure S1), can be regarded as pathogenic. However, due to its localization (terminal from 3326 position), it is usually considered as a benign variant. Indeed, in ClinVar database, 11 of the 15 entries interpreted this variant as benign/likely benign and four submitters considered it as a variant with unknown significance (VUS). Additionally, of the 11 studies reporting c.10095delins GAATTATATCT, the vast majority considered it as a VUS [18-20] or clinically not important [21] in breast/ovarian cancer patients, and a VUS in familial pancreatic cancer [22], while it was interpreted as benign in ovarian cancer patients [23]. Interestingly, in a study prioritizing variants in hereditary breast and ovarian cancer genes in patients lacking known BRCA mutations, the c.10095delins GAATTATATCT 
variant was categorized as likely pathogenic based on co-segregation analysis (likelihood ratio 3.71 ) [24].

Therefore, the aim of our study was to investigate the prevalence of BRCA2 C-terminal stop codon variants among our breast/ovarian cancer patients sent to germline $B R C A 1 / 2$ gene testing and their co-segregation with clinicopathological parameters and study the loss of heterozygosity and allelic imbalance. An extensive literature review of an additional 122,209 cancer patients was also performed to assess the effects of the c.9976A $>$ T variant on cancer risk.

\section{Results}

2.1. Frequency and Characteristics of BRCA2 Terminal Stop Codon Variants in Breast Cancer Patients

Out of 2491 independent breast/ovarian cancer patients, c.9976A > T and/or c.10095delins GAATTATATCT stop variants were identified in 49 cases (Figure 1). Among 49 cases, c.9976A $>$ T was detected in 36, c.10095delinsGAATTATATCT in 12 cases and c.9976A $>$ T together with c.10095delinsGAATTATATCT in 1 case. These variants co-occurred with other pathogenic $B R C A 1$ or $B R C A 2$ variants (c.9976A $>\mathrm{T}$ in five cases (5/37: $13.51 \%$ ) and c.10095delinsGAATTATATCT in two cases (2/13: 15.38\%)) (Table 1). The frequency of double heterozygosity in the investigated population was low: 0.002 (5/2491) and 0.0008 (2/2491) for c.9976A>T and c.10095delinsGAATTATATCT, respectively. Pathogenic BRCA1 with a pathogenic $B R C A 2$ variant in the same patient has not been identified in our cohort (Figure 1).

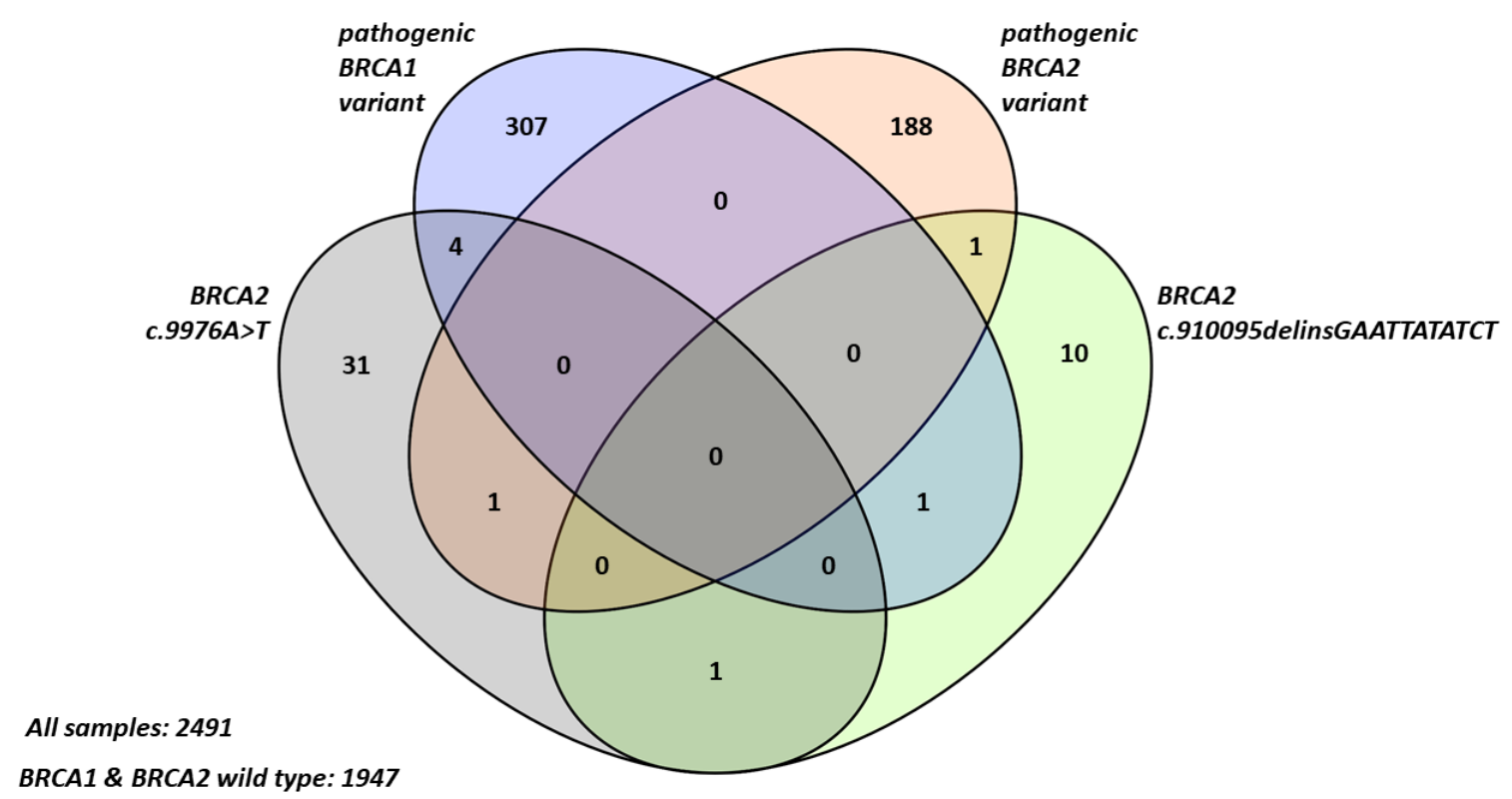

Figure 1. Numbers of $B R C A 1$ and $B R C A 2$ variants identified in our cohort.

Age of disease appearance did not differ in terminal stop codon variant carriers compared to pathogenic BRCA2 carriers or $B R C A 1 / 2$ wild type patients (see Table 2 ). The Ki67\% proliferation index, the prevalence of triple negative breast cancer (TNBC) or multiplex HBOC were increased only when the c.9976A $>$ T variant accompanied a pathogenic BRCA1 variant (Table 2). 
Table 1. Patient characteristics harboring BRCA2 C-terminal variants (LRG_292t1:c.9976A>T, p.(Lys3326Ter) and LRG_292t1:c.10095delinsGAATTATATCT, p.(Ser3366AsnfsTer4)).

\begin{tabular}{|c|c|c|c|c|c|c|c|c|c|c|c|c|c|}
\hline \multirow{3}{*}{$\#$} & \multirow{3}{*}{ Gender } & \multirow{3}{*}{$\begin{array}{c}\text { BRCA2 C- } \\
\text { Terminal } \\
\text { Variant }\end{array}$} & \multirow{3}{*}{$\begin{array}{c}\text { Pathogenic } \\
\text { BRCA1/BRCA2 } \\
\text { Variant }\end{array}$} & \multirow{3}{*}{ Disease: } & \multicolumn{9}{|c|}{ Proband } \\
\hline & & & & & \multicolumn{6}{|c|}{ 1st Breast Cancer } & \multirow{2}{*}{$\begin{array}{c}\begin{array}{c}\text { 2nd } \\
\text { Breast } \\
\text { Cancer }\end{array} \\
\text { Age }\end{array}$} & \multicolumn{2}{|c|}{ Ovarian Cancer } \\
\hline & & & & & $\begin{array}{c}\text { Age of } \\
\text { onset }\end{array}$ & ER & PR & HER2 & Ki67 (\%) & Hist & & Age & Hist \\
\hline 1 & $\mathrm{~F}$ & c. $9976 \mathrm{~A}>\mathrm{T}$ & - & sol & 43 & pos & pos & neg & 25 & DUC & - & - & - \\
\hline 2 & $\mathrm{~F}$ & c. $9976 \mathrm{~A}>\mathrm{T}$ & - & sol & 55 & pos & neg & neg & n.a. & DUC & - & - & - \\
\hline 3 & $\mathrm{~F}$ & c. $9976 \mathrm{~A}>\mathrm{T}$ & - & sol & 48 & pos & pos & neg & n.a. & DUC & - & - & - \\
\hline 4 & $\mathrm{~F}$ & c. $9976 \mathrm{~A}>\mathrm{T}$ & - & sol & 47 & pos & pos & neg & 5 & DUC & - & - & - \\
\hline 5 & $\mathrm{~F}$ & c. $9976 \mathrm{~A}>\mathrm{T}$ & - & sol & 31 & pos & pos & neg & 40 & DUC & - & - & - \\
\hline 6 & $\mathrm{~F}$ & c. $9976 \mathrm{~A}>\mathrm{T}$ & - & sol & 68 & pos & pos & neg & 1 & DUC & - & - & - \\
\hline 7 & $\mathrm{~F}$ & c. $9976 \mathrm{~A}>\mathrm{T}$ & - & sol & 57 & pos & pos & neg & 10 & DUC & - & - & - \\
\hline 8 & $\mathrm{~F}$ & c. $9976 \mathrm{~A}>\mathrm{T}$ & - & sol & 36 & neg & neg & neg & 40 & DUC & - & - & - \\
\hline 9 & $\mathrm{~F}$ & c. $9976 \mathrm{~A}>\mathrm{T}$ & - & sol & 42 & pos & pos & neg & 70 & LOB & - & - & - \\
\hline 10 & $\mathrm{~F}$ & c. $9976 \mathrm{~A}>\mathrm{T}$ & - & sol & 46 & pos & pos & neg & n.a. & DUC & - & - & - \\
\hline 11 & $\mathrm{~F}$ & c. $9976 \mathrm{~A}>\mathrm{T}$ & - & sol & 41 & pos & pos & pos & 10 & DUC & - & - & - \\
\hline 12 & $\mathrm{~F}$ & c. $9976 \mathrm{~A}>\mathrm{T}$ & - & sol & 34 & pos & pos & pos & 67 & DUC & - & - & - \\
\hline 13 & $\mathrm{~F}$ & c. $9976 \mathrm{~A}>\mathrm{T}$ & - & sol & 39 & neg & neg & neg & 20 & DUC & - & - & - \\
\hline 14 & $\mathrm{~F}$ & c. $9976 \mathrm{~A}>\mathrm{T}$ & - & sol & 46 & pos & pos & neg & 5 & DUC & - & - & - \\
\hline 15 & $\mathrm{~F}$ & c. $9976 \mathrm{~A}>\mathrm{T}$ & - & sol & 41 & pos & neg & neg & 25 & DUC & - & - & - \\
\hline 16 & $\mathrm{~F}$ & c.9976A > T & - & sol & 46 & pos & pos & neg & n.a. & DUC & - & - & - \\
\hline 17 & $\mathrm{~F}$ & c. $9976 \mathrm{~A}>\mathrm{T}$ & - & sol & 44 & pos & pos & neg & n.a. & DUC & - & - & - \\
\hline 18 & $\mathrm{~F}$ & c. $9976 \mathrm{~A}>\mathrm{T}$ & - & sol & 38 & pos & pos & neg & 25 & DUC & - & - & - \\
\hline 19 & $\mathrm{~F}$ & c. $9976 \mathrm{~A}>\mathrm{T}$ & - & multi & 33 & pos & pos & neg & 50 & DUC & - & 33 & $\begin{array}{c}\text { adenocar- } \\
\text { cinoma }\end{array}$ \\
\hline 20 & $\mathrm{~F}$ & c.9976A>T & - & sol & 33 & pos & pos & neg & 25 & DUC & - & - & - \\
\hline
\end{tabular}


Table 1. Cont.

\begin{tabular}{|c|c|c|c|c|c|c|c|c|c|c|c|c|c|}
\hline \multirow{3}{*}{$\#$} & \multirow{3}{*}{ Gender } & \multirow{3}{*}{$\begin{array}{c}\text { BRCA2 C-Terminal } \\
\text { Variant }\end{array}$} & \multirow{3}{*}{$\begin{array}{l}\text { Pathogenic } \\
\text { BRCA1/BRCA2 } \\
\text { Variant }\end{array}$} & \multirow{3}{*}{ Disease: } & \multicolumn{9}{|c|}{ Proband } \\
\hline & & & & & \multicolumn{6}{|c|}{ 1st Breast Cancer } & \multirow{2}{*}{$\begin{array}{c}\begin{array}{c}\text { 2nd } \\
\text { Breast } \\
\text { Cancer }\end{array} \\
\text { Age }\end{array}$} & \multicolumn{2}{|c|}{ Ovarian Cancer } \\
\hline & & & & & $\begin{array}{c}\text { Age of } \\
\text { onset }\end{array}$ & ER & PR & HER2 & Ki67 (\%) & Hist & & Age & Hist \\
\hline 21 & $\mathrm{~F}$ & c. $9976 \mathrm{~A}>\mathrm{T}$ & - & sol & 61 & neg & neg & neg & 25 & DUC & - & - & - \\
\hline 22 & $\mathrm{~F}$ & c. $9976 \mathrm{~A}>\mathrm{T}$ & - & sol & 46 & neg & neg & poz & 10 & DUC & - & - & - \\
\hline 23 & $\mathrm{~F}$ & c. $9976 \mathrm{~A}>\mathrm{T}$ & - & sol & 40 & pos & neg & neg & 70 & DUC & - & - & - \\
\hline 24 & $\mathrm{~F}$ & c. $9976 \mathrm{~A}>\mathrm{T}$ & - & multi & 48 & pos & n.a. & neg & n.a. & DUC & 48 & - & - \\
\hline 25 & $\mathrm{~F}$ & c. $9976 \mathrm{~A}>\mathrm{T}$ & - & sol & 26 & pos & pos & pos & 35 & DUC & - & - & - \\
\hline 26 & $\mathrm{~F}$ & c. $9976 \mathrm{~A}>\mathrm{T}$ & - & sol & 41 & pos & pos & neg & n.a. & DUC & - & - & - \\
\hline 27 & $\mathrm{~F}$ & c. $9976 \mathrm{~A}>\mathrm{T}$ & - & sol & 44 & pos & pos & neg & 25 & DUC & - & - & - \\
\hline 28 & $\mathrm{~F}$ & c. $9976 \mathrm{~A}>\mathrm{T}$ & - & sol & 33 & pos & pos & neg & 35 & DUC & - & - & - \\
\hline 29 & $\mathrm{~F}$ & c. $9976 \mathrm{~A}>\mathrm{T}$ & - & sol & 43 & pos & pos & neg & 7 & DUC & - & - & - \\
\hline 31 & $\mathrm{~F}$ & c. $9976 \mathrm{~A}>\mathrm{T}$ & - & sol & n.a. & n.a. & n.a. & n.a. & n.a. & n.a. & - & 45 & $\begin{array}{c}\text { cystadenoc } \\
\text { arcinoma } \\
\text { muci- } \\
\text { nosum }\end{array}$ \\
\hline 32 & $\mathrm{~F}$ & c.10095delinsGAATTATATCT & - & sol & 44 & pos & pos & neg & 10 & DUC & - & - & - \\
\hline 33 & $\mathrm{~F}$ & c.10095delinsGAATTATATCT & - & sol & 36 & neg & neg & neg & 30 & DUC & - & - & - \\
\hline 34 & $\mathrm{~F}$ & c.10095delinsGAATTATATCT & - & sol & 55 & neg & neg & neg & 50 & DUC & - & - & - \\
\hline 35 & $\mathrm{~F}$ & c.10095delinsGAATTATATCT & - & sol & 55 & n.a. & n.a. & n.a. & n.a. & n.a. & - & - & - \\
\hline 36 & $\mathrm{~F}$ & c.10095delinsGAATTATATCT & - & sol & 40 & pos & pos & neg & 15 & DUC & - & - & - \\
\hline 37 & $\mathrm{~F}$ & c.10095delinsGAATTATATCT & - & sol & 27 & pos & pos & neg & n.a. & LOB & - & - & - \\
\hline 38 & $\mathrm{~F}$ & c.10095delinsGAATTATATCT & - & sol & 39 & pos & pos & neg & 10 & LOB & - & - & - \\
\hline 39 & $\mathrm{~F}$ & c.10095delinsGAATTATATCT & - & sol & 45 & pos & pos & neg & 1 & DUC & - & - & - \\
\hline
\end{tabular}


Table 1. Cont.

\begin{tabular}{|c|c|c|c|c|c|c|c|c|c|c|c|c|c|}
\hline \multirow{3}{*}{$\#$} & \multirow{3}{*}{ Gender } & \multirow{3}{*}{$B R C A 2$ C-Terminal Variant } & \multirow{3}{*}{$\begin{array}{c}\text { Pathogenic } \\
\text { BRCA1/BRCA2 } \\
\text { Variant }\end{array}$} & \multirow{3}{*}{ Disease: } & \multicolumn{9}{|c|}{ Proband } \\
\hline & & & & & \multicolumn{6}{|c|}{ 1st Breast Cancer } & \multirow{2}{*}{$\begin{array}{c}\begin{array}{c}\text { 2nd } \\
\text { Breast } \\
\text { Cancer }\end{array} \\
\text { Age }\end{array}$} & \multicolumn{2}{|c|}{ Ovarian Cancer } \\
\hline & & & & & $\begin{array}{c}\text { Age of } \\
\text { onset }\end{array}$ & ER & PR & HER2 & Ki67 (\%) & Hist & & Age & Hist \\
\hline 40 & $\mathrm{~F}$ & c.10095delinsGAATTATATCT & - & sol & 38 & pos & pos & neg & 2 & DUC & - & - & - \\
\hline 41 & $\mathrm{~F}$ & c.10095delinsGAATTATATCT & - & multi & 55 & pos & pos & neg & 20 & LOB & 58 & - & - \\
\hline 42 & $\mathrm{~F}$ & $\begin{array}{c}\text { c.9976A }>\text { T \& } \\
\text { c.10095delinsGAATTATATCT }\end{array}$ & - & sol & 41 & pos & pos & neg & n.a. & DUC & - & - & - \\
\hline 43 & $\mathrm{~F}$ & c.10095delinsGAATTATATCT & $\begin{array}{c}\text { BRCA1 c. } 5251 \mathrm{C}>\mathrm{T} \\
\quad(\text { p.Arg1751*) }\end{array}$ & sol & n.a. & n.a. & n.a. & n.a. & n.a. & n.a. & - & 36 & $\begin{array}{l}\text { high grade } \\
\text { serosus } \\
\text { carcinoma }\end{array}$ \\
\hline 44 & $\mathrm{~F}$ & c. $9976 \mathrm{~A}>\mathrm{T}$ & $\begin{array}{l}\text { BRCA1 c. } 1687 \mathrm{C}>\mathrm{T} \\
(\text { p.Gln563*) }\end{array}$ & sol & 40 & pos & pos & pos & 50 & DUC & - & - & - \\
\hline 45 & $\mathrm{~F}$ & c. $9976 \mathrm{~A}>\mathrm{T}$ & $\begin{array}{c}\text { BRCA1 } \\
\text { c.68_69delAG } \\
\text { (p.Glu23Valfs*) }\end{array}$ & sol & 54 & neg & neg & neg & 85 & DUC & - & - & - \\
\hline 46 & $\mathrm{~F}$ & c. $9976 \mathrm{~A}>\mathrm{T}$ & $\begin{array}{c}\text { BRCA1 } \\
\text { c.3018_3021del4 } \\
\text { (p.His1006Glnfs*17) }\end{array}$ & sol & 38 & n.a. & n.a. & n.a. & n.a. & n.a. & - & - & - \\
\hline 47 & $\mathrm{~F}$ & c. $9976 \mathrm{~A}>\mathrm{T}$ & $\begin{array}{c}\text { BRCA1 c. } 181 \mathrm{~T}>\mathrm{G} \\
\text { (p.Cys61Gly) }\end{array}$ & sol & 49 & neg & neg & neg & 90 & DUC & - & - & - \\
\hline 48 & M & c. $9976 \mathrm{~A}>\mathrm{T}$ & $\begin{array}{c}\text { BRCA2 } \\
\text { c.8378G }>A \\
\text { (p.Gly2793Glu) }\end{array}$ & sol & 79 & pos & pos & neg & 25 & DUC & - & - & - \\
\hline 49 & $\mathrm{~F}$ & c.10095delinsGAATTATATCT & $\begin{array}{c}\text { BRCA2 } \\
\text { c.7595_7596insTT } \\
\text { (p.Ala2534Leufs*18) }\end{array}$ & sol & 38 & poz & poz & n.a. & 5 & DUC & - & - & - \\
\hline
\end{tabular}

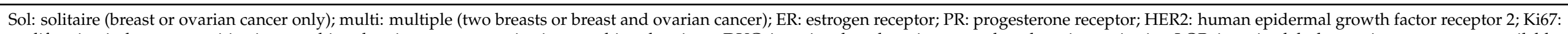

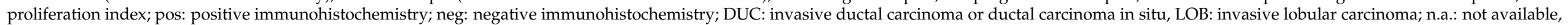
Hist: histology. 
Table 2. Cancer prevalence and tumor characteristics in hereditary breast and ovarian cancer (HBOC) probands and their families (proportions with $\pm 95 \% \mathrm{CI}$ ).

\begin{tabular}{|c|c|c|c|c|c|c|}
\hline $\begin{array}{l}\text { Clinicopathological } \\
\text { Parameter }\end{array}$ & BRCA1/2 Wild Type & $\begin{array}{c}\text { Pathogenic } B R C A 1 \\
\text { Variant }\end{array}$ & $\begin{array}{c}\text { Pathogenic } B R C A 2 \\
\text { Variant }\end{array}$ & $B R C A 2$ c. $9976 \mathrm{~A}>\mathrm{T}$ & $\begin{array}{c}\text { BRCA2 c.10095delins } \\
\text { GAATTATATCT }\end{array}$ & $\begin{array}{c}\text { Pathogenic } B R C A 1+ \\
B R C A 2 \text { c. } 9976 \mathrm{~A}>\mathrm{T}\end{array}$ \\
\hline Number of probands $(n)$ & 1947 & 307 & 188 & 31 & 10 & 4 \\
\hline \multicolumn{7}{|c|}{ Age at disease onset (years): } \\
\hline $\begin{array}{l}\text { Breast cancer (mean } \pm \\
\text { SD) }\end{array}$ & $43.38 \pm 9.33$ & $39.48 \pm 8.93 \mathbf{a}$ & $41.93 \pm 8.63 \mathbf{b}$ & $43.1 \pm 9.10 \mathbf{b}$ & $43.4 \pm 9.37$ & $42.25 \pm 7.54$ \\
\hline $\begin{array}{l}\text { Ovarian cancer (mean } \pm \\
\text { SD) }\end{array}$ & $49.07 \pm 13.85$ & $48.41 \pm 7.99$ & $55.3 \pm 10.03$ & 45.00 & - & - \\
\hline $\begin{array}{l}\text { Male breast cancer } \\
\quad(\text { mean } \pm \mathrm{SD})\end{array}$ & $59.74 \pm 11.08$ & 47.00 & $62.87 \pm 8.82$ & 27.00 & - & - \\
\hline $\begin{array}{l}\text { Multiplex tumors from } \\
\text { all patients (proportion } \\
(\mathrm{mpx} \text { cases } / \text { all }))\end{array}$ & $0.08(156 / 1947)$ & $0.2(60 / 307) \mathbf{a}$ & $0.18(34 / 188) \mathbf{a}$ & $0.06(2 / 31)$ & $0.10(1 / 10)$ & $0.00(0 / 4)$ \\
\hline \multicolumn{7}{|l|}{ Tumor characteristics: } \\
\hline $\begin{array}{l}\text { Ki67 of breast cancer } \\
(\text { mean } \pm \mathrm{SD})\end{array}$ & $30 \pm 25$ & $58 \pm 24 \mathbf{a}$ & $30 \pm 24$ & $28 \pm 21$ & $17 \pm 16$ & $75 \pm 22$ \\
\hline $\begin{array}{l}\text { PR pos proportion } \\
(95 \% \mathrm{CI})\end{array}$ & $0.64(0.62-0.66)$ & $0.15(0.11-0.20) \mathbf{a}$ & $0.67(0.60-0.74) \mathbf{b}$ & $0.75(0.56-0.87)$ & $0.77(0.44-0.94)$ & $0.33(0.05-0.79)$ \\
\hline $\begin{array}{l}\text { HER2 pos proportion } \\
(95 \% \mathrm{CI})\end{array}$ & $0.24(0.22-0.26)$ & $0.06(0.04-0.11) \mathbf{a}$ & $0.10(0.06-0.16) \mathbf{a}$ & $0.13(0.05-0.31)$ & $0(0.00-0.34)$ & $0.33(0.05-0.79)$ \\
\hline $\begin{array}{l}\text { TNBC proportion } \\
(95 \% \mathrm{CI})\end{array}$ & $0.20(0.19-0.23)$ & $0.75(0.70-0.80) \mathbf{a}$ & $0.19(0.14-0.26) \mathbf{b}$ & $0.10(0.03-0.27) \mathbf{b}$ & $0.22(0.05-0.55)$ & $0.66(0.20-0.94)$ \\
\hline \multicolumn{7}{|c|}{ Tumor prevalence in families (proportion $(95 \% \mathrm{CI})$ ) } \\
\hline $\begin{array}{c}\text { Breast cancer }<50 \text { years } \\
\text { of age in the family }\end{array}$ & $0.10(0.09-0.11)$ & $0.21(0.16-0.25)$ a & $0.24(0.18-0.30)$ a & $0.16(0.06-0.33)$ & $0.10(0.00-0.42)$ & $0(0.00-0.54)$ \\
\hline $\begin{array}{l}\text { Breast cancer at any age } \\
\text { in the family }\end{array}$ & $0.42(0.40-0.44)$ & $0.61(0.56-0.66) \mathbf{a}$ & $0.60(0.53-0.67) \mathbf{a}$ & $0.38(0.24-0.56) \mathbf{b}, \mathbf{c}$ & $0.5(0.24-0.76)$ & $0.25(0.03-0.71)$ \\
\hline $\begin{array}{l}\text { Ovarian cancer at any } \\
\text { age in the family }\end{array}$ & $0.06(0.05-0.08)$ & $0.21(0.17-0.26) \mathbf{a}$ & $0.08(0.05-0.13) \mathbf{b}$ & $0.06(0.01-0.21)$ & $0(0.00-0.32)$ & $0.25(0.03-0.71)$ \\
\hline
\end{tabular}


Table 2. Cont.

\begin{tabular}{|c|c|c|c|c|c|c|}
\hline $\begin{array}{c}\text { Clinicopathological } \\
\text { Parameter }\end{array}$ & BRCA1/2 Wild Type & $\begin{array}{c}\text { Pathogenic BRCA1 } \\
\text { Variant }\end{array}$ & $\begin{array}{c}\text { Pathogenic } B R C A 2 \\
\text { Variant }\end{array}$ & $B R C A 2$ c.9976A $>\mathrm{T}$ & $\begin{array}{c}\text { BRCA2 c.10095delins } \\
\text { GAATTATATCT }\end{array}$ & $\begin{array}{c}\text { Pathogenic } B R C A 1+ \\
B R C A 2 \text { c.9976A }>\mathrm{T}\end{array}$ \\
\hline $\begin{array}{c}\text { Breast and/or ovarian } \\
\text { cancer at any age in the } \\
\text { family }\end{array}$ & $0.47(0.44-0.49)$ & $0.69(0.63-0.74) \mathbf{a}$ & $0.65(0.58-0.72) \mathbf{a}$ & $0.45(0.29-0.62) \mathbf{b}, \mathbf{c}$ & $0.5(0.23-0.76)$ & $0.5(0.15-0.85)$ \\
\hline $\begin{array}{l}\text { Prostate cancer in the } \\
\text { family }\end{array}$ & $0.08(0.07-0.09)$ & $0.04(0.03-0.07) \mathbf{a}$ & $0.11(0.07-0.17) \mathbf{b}$ & $0.06(0.01-0.21)$ & $0.20(0.05-0.52)$ & $0.25(0.03-0.71)$ \\
\hline $\begin{array}{l}\text { Pancreatic cancer in the } \\
\text { family }\end{array}$ & $0.04(0.03-0.05)$ & $0.06(0.04-0.09)$ & $0.10(0.06-0.15) \mathbf{a}$ & $0.09(0.03-0.25)$ & $0(0.00-0.32)$ & $0.5(0.15-0.85) \mathbf{a}, \mathbf{b}$ \\
\hline $\begin{array}{l}\text { Lung cancer in the } \\
\text { family }\end{array}$ & $0.13(0.12-0.15)$ & $0.09(0.06-0.13)$ & $0.09(0.05-0.14)$ & $0.22(0.11-0.40) \quad b, c$ & $0(0.00-0.32)$ & $0(0.00-0.54)$ \\
\hline Skin cancer in the family & $0.04(0.03-0.05)$ & $0.03(0.02-0.06)$ & $0.03(0.01-0.07)$ & $0.09(0.02-0.25)$ & $0.10(0.00-0.42)$ & $0(0.00-0.54)$ \\
\hline $\begin{array}{l}\text { Head and neck cancer in } \\
\text { the family }\end{array}$ & $0.05(0.04-0.06)$ & $0.05(0.03-0.08)$ & $0.08(0.05-0.13)$ & $0(0.00-0.13)$ & $0(0.00-0.32)$ & $0(0.00-0.54)$ \\
\hline $\begin{array}{l}\text { Hepatobiliary cancer in } \\
\text { the family }\end{array}$ & $0.03(0.02-0.04)$ & $0.03(0.01-0.05)$ & $0.04(0.02-0.08)$ & $0(0.00-0.13)$ & $0(0.00-0.32)$ & $0.25(0.03-0.71)$ \\
\hline
\end{tabular}

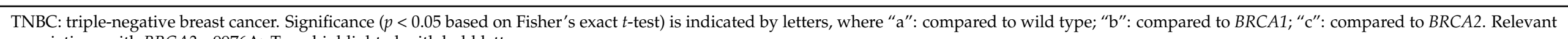
associations with BRCA2 c.9976A $>$ T are highlighted with bold letters. 


\subsection{Familial Cancer Prevalence in Probands with BRCA2 Terminal Stop Codon Variants}

\subsubsection{Hereditary Breast and Ovarian Cancer (HBOC) Syndrome-Related Cancers}

To assess the effects of the c.9976A > T and c.10095delinsGAATTATATCT variants on cancer risk, standard familial data were obtained from all probands (see Methods). Accordingly, "strong familiarity" was defined by the presence of breast/ovarian cancer before 50 years and/or male breast cancer in the family. Additionally, cases were categorized as "syndromic" when HBOC-related tumors (breast, ovarian, male breast, prostate or pancreatic cancer) occurred in the family of the proband (Table S1).

Expectedly, regarding HBOC-related tumors in the family of probands carrying a pathogenic $B R C A 1 / 2$ variant, breast and ovarian cancer were more frequent before the age of 50 years, and irrespective of age as well. The prevalence of HBOC-related tumors did not differ in families of c.9976A $>$ T or c.10095delinsGAATTATATCT carrier probands as compared to BRCA1/2 wild type patients (Table 2).

Pancreas and prostate cancers were more common in families with pathogenic $B R C A 2$ variant carrier probands (Table 2, Figure 2). Interestingly, pancreas cancer was also more frequent in double heterozygotes of a $B R C A 1$ pathogenic variant and $B R C A 2 \mathrm{c} .9976 \mathrm{~A}>\mathrm{T}$ variant, suggesting a potential genetic modifier effect (Table 2, Figure 2). The statistical power of this comparison was $78.3 \%$.
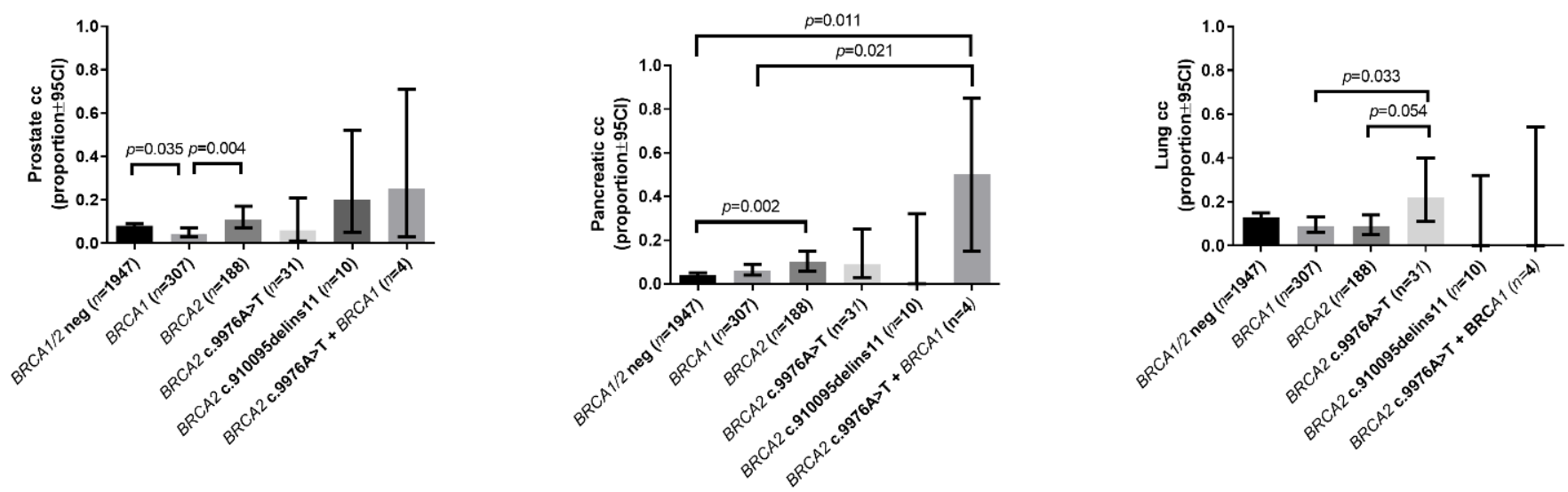

Figure 2. Prostate, pancreatic and lung cancer prevalence in HBOC probands' families (proportions with $\pm 95 \% \mathrm{CI}$ ).

Strong familiarity and syndromic familial history were characteristic only for the families of pathogenic BRCA1/2 variant carriers, while families of probands carrying only the $B R C A 2$ C-terminal stop codon did not differ from $B R C A 1 / 2$ wild type patients' families regarding HBOC syndrome-related tumor types (breast, ovarian, prostate and pancreatic cancer) (Table 2). In addition, pancreatic cancer occurred more frequently in families carrying a pathogenic $B R C A 1$ variant along with $B R C A 2$ c.9976A $>$ T (double heterozygotes) (0.5) compared to wild type (0.04) and pathogenic BRCA1 carrier families (0.06) (Table 2, Figure 2).

\subsubsection{Prevalence of Other Cancers}

We assessed the frequency of lung, skin, head and neck, hepatocellular and gastric cancer in our cohort. We observed that lung cancer was more common in families of $B R C A 2$ c.9976A > T carrier probands (0.22) when compared to BRCA1/2 wild type (0.13) or pathogenic BRCA1 (0.09) or BRCA2 (0.09) variant carrier families (Table 2, Figure 2). We did not find significant differences in the occurrence of head and neck, gastric or hepatocellular cancer in our families (Table 2, Table S2). 
2.3. Functional Evaluation of the Potential Pathogenicity of BRCA2 C-Terminal Stop Codon Variants (Loss Of Heterozygosity, Allelic Imbalance, Minor Allele Frequency)

Loss of heterozygosity $(\mathrm{LOH})$ in the tumor sample of a variant carrier is considered as supporting evidence for pathogenicity according to the ACMG guidelines. Therefore, we tested $\mathrm{LOH}$ in c.9976A $>$ T carrier cases where c.9976A $>$ T occurred without any other pathogenic $B R C A 1$ or $B R C A 2$ variant and where a tumor sample was available. In 26 tumor tissue-blood (somatic vs. germline) pairs, we did not confirm LOH in any of the tumor specimens.

We also investigated if C-terminal stop codon variants influence allelic stability in three samples. By cDNA sequencing, we did not find difference in allelic expression between wild type and variant-carrier strands in c.9976A > T nor c.10095delinsGAATTATATCT cases.

Variant segregation with disease phenotype supports pathogenicity. As, currently, c.9976A > T and c.10095delinsGAATTATATCT are not considered obviously pathogenic variants for HBOC cancers, healthy family members could not be screened for this variant within our national genetic counseling system.

Regarding allelic frequency, we observed a minor allele frequency (MAF) of c.9976A > T as 0.0074 in our proband population that did not differ significantly from those reported for European non-Finnish samples or total MAFs (0.008723 and 0.006468 , respectively) based on the gnomAD database.

Determining the prevalence of BRCA2 c.10095delinsGAATTATATCT was challenging because it is a complex variant (a combination of a deletion and insertion), therefore, in different databases, it appears as two distinct variants (e.g., in the gnomAD database: c.10094_10095insGAATTATAT and c.10095_10096insT, leading to frameshift and a premature stop codon), due to different variant-calling algorithms used during variant annotation of next generation sequencing data. (The correct description of the variant is c.10095delinsGAATTATATCT according to Human Genome Variation Society (HGVS) nomenclature.) Accordingly, we found that the BRCA2 c.10095delinsGAATTATATCT variant was more prevalent among our patients compared to the control population $(0.00261$ vs. 0.00047, respectively).

Recently, Higgs et al. reported multiple co-occurrences of the BRCA2 c.9976A>T variant with the pathogenic BRCA2 c.6275_6276delTT (p.(Leu2092ProfsTer7)) frameshift variant in 52 families, while only $1.3-1.7 \%$ of the patients carried the BRCA2 c.9976A>T variant alone [7]. Therefore, we investigated if these variants are in linkage in our patient cohort. Surprisingly, we did not detect the BRCA2 c.6275_6276delTT variant in our sample set (2491 probands) at all, including all patients carrying BRCA2 c.9976A > T (Table 1).

\subsection{Re-Analysis of BRCA2 c.9976A>T and c.10095delinsGAATTATATCT Variants by Re-Analysis of All Published Data Where These Variants Were Investigated}

Due to its indistinct annotation, still, there are literature data regarding the $B R C A 2$ c.10095delinsGAATTATATCT variant (Table 3). Allelic frequency is observed in a wide range (0.00099-0.03846), but in all data sets, it was less than 0.04. Most of the reports interpreted the variant as having unknown significance, but in ovarian cancer, it was described as a benign variant (Table 3 ). 
Table 3. Prevalence of BRCA2 c.10095delinsGAATTATATCT based on literature data.

\begin{tabular}{|c|c|c|c|c|c|}
\hline Cancer Type & Reference & $\begin{array}{l}\text { Number of } \\
\text { Probands Screened } \\
\text { (Germline) }\end{array}$ & $\begin{array}{c}\text { Number of Patients } \\
\text { Carrying } B R C A 2 \\
\text { c.10095delins } \\
\text { GAATTATATCT } \\
\text { Variant }\end{array}$ & Allelic Frequency & $\begin{array}{c}\text { Clinical } \\
\text { Interpretation }\end{array}$ \\
\hline breast, ovarian cancer & $\begin{array}{c}\text { Meindl et al. } 2002 \\
\text { [20] }\end{array}$ & 989 & 3 & 0.00303 & VUS \\
\hline breast, ovarian cancer & $\begin{array}{c}\text { Ratajska et al. } 2008 \\
\text { [18] }\end{array}$ & 64 & 2 & 0.03125 & VUS \\
\hline breast, ovarian cancer & $\begin{array}{c}\text { Machackova et al. } \\
2008 \text { [19] }\end{array}$ & 1010 & 1 & 0.00099 & VUS \\
\hline breast, ovarian cancer & Cvok et al. 2008 [21] & 115 & 1 & 0.00869 & $\begin{array}{l}\text { clinically not } \\
\text { important }\end{array}$ \\
\hline breast, ovarian cancer & $\begin{array}{l}\text { Thomassen et al. } \\
2008^{* *}[25]\end{array}$ & na & 1 & na & not interpreted \\
\hline breast, ovarian cancer & $\begin{array}{c}\text { Meisel et al. } 2017^{* *,+} \\
{[26]}\end{array}$ & 523 & 3 & 0.00573 & VUS \\
\hline ovarian cancer & $\begin{array}{c}\text { Koczkowska et al. } \\
2016 \text { [23] }\end{array}$ & $22^{*}$ & 1 & na * & benign \\
\hline pancreatic cancer & Hahn et al. 2013 [22] & 26 & 1 & 0.03846 & VUS \\
\hline
\end{tabular}

Because of the controversial literature data regarding the clinical relevance of the $B R C A 2$ c.9976A $>$ T variant, we conducted an extensive literature search and collected all available data. We found 38 studies reporting 122,209 cases investigating BRCA2 gene variants in different cancer types including breast, ovarian, pancreatic, lung, upper aerodigestive system, urinary tract and skin cancers (Table 4). BRCA2 c.9976A >T was available for evaluation in 115,854 cases. Carrier status was reported in 5129 patients of the 115,854 cancer cases. The average minor allele frequency (MAF) of the variant in breast/ovarian cancer patients was 0.0096. Regarding breast cancer cases, the average MAF was 0.0093. In terms of breast and ovarian cancer, odds ratios (ORs) were 0.41-1.53 (Table 4). Among studies investigating only ovarian cancer patients, the ORs were found to be similar, only Stafford et al. (2017) reported a significantly higher OR (OR: 4.95; $p=0.01$; four of 48) [16]. In familial pancreatic cancer, lung cancer and upper aero-digestive tract (UADT) cancer, the carrier status meant a high odds ratio (4.24, 3 and 2.53, respectively) for developing cancer $[8,10,17]$. In the study of Akbari et al. (2008), c.9976A $>$ T carrier status was associated with a high OR for developing esophageal squamous cell carcinoma (6.0; 95\%CI: 1.3-28; $p=0.01$ ) [27]. In other studies, BRCA2 c.9976A $>\mathrm{T}$ carrier status was associated with a moderate risk for cancer (Table 4). 
Table 4. Prevalence and association of $B R C A 2$ c.9976A $>$ T variant with various cancers in 38 studies.

\begin{tabular}{|c|c|c|c|c|c|}
\hline Cancer Type & Reference & $\begin{array}{c}\text { Number of } \\
\text { Probands Screened }\end{array}$ & $\begin{array}{c}\text { Number of Patients } \\
\text { Carrying } B R C A 2 \\
\text { c.9976A }>\text { T Variant }\end{array}$ & Allelic Frequency & $\begin{array}{c}\text { Odds Ratio (OR) } \\
\text { (Patients vs. } \\
\text { Controls) } \\
\text { (Confidence } \\
\text { Intervals) }\end{array}$ \\
\hline breast, ovarian cancer & current study & 2138 & 46 & 0.01485 & na \\
\hline breast cancer & $\begin{array}{c}\text { Mazoyer et al. } 1996 \\
{[11]}\end{array}$ & 513 & 11 & 0.01267 & OR: 1.01 (0.41-2.48) \\
\hline breast cancer & $\begin{array}{l}\text { Johnson et al. } 2007 \\
\text { [12] }\end{array}$ & 473 & 11 & 0.011628 & OR: 1.16 (0.79-1.63) \\
\hline breast cancer & Borg et al. 2010 [28] & 2103 & 40 & 0.00951 & na \\
\hline breast cancer & $\begin{array}{l}\text { Michailidou et al. } \\
2013 \text { [14] }\end{array}$ & 10052 & 80 & 0.008 & $\begin{array}{l}\text { RR: } 1.391 .39 \\
(1.13-1.71)\end{array}$ \\
\hline breast cancer & $\begin{array}{c}\text { Thompson et al. } 2015 \\
\text { [9] }\end{array}$ & 2634 & 66 & 0.01252 & $\begin{array}{c}\text { OR: } 1.53(1.00-2.34) \\
(p=0.047)\end{array}$ \\
\hline breast cancer & Meeks et al. 2016 [15] & 41081 & 852 & 0.01036 & $\begin{array}{l}\text { OR: } 1.28(1.17-1.40) \\
\quad\left(p=5.86 \times 10^{-6}\right)\end{array}$ \\
\hline $\begin{array}{l}\text { breast cancer, early } \\
\text { onset }\end{array}$ & $\begin{array}{c}\text { Krainer et al.1. } 1997 \\
\text { [29] }\end{array}$ & 73 & 1 & 0.00684 & na \\
\hline $\begin{array}{l}\text { breast cancer, early } \\
\text { onset }\end{array}$ & $\begin{array}{c}\text { Malone et al. } 2000 \\
{[30]}\end{array}$ & 386 & 2 & 0.00259 & na \\
\hline $\begin{array}{l}\text { breast cancer, early } \\
\text { onset }\end{array}$ & $\begin{array}{l}\text { Bergthorsson et al. } \\
\text { 2001 [31] }\end{array}$ & 119 & 1 & 0.00420 & na \\
\hline $\begin{array}{l}\text { breast cancer, early } \\
\text { onset }\end{array}$ & $\begin{array}{l}\text { Hamann et al. } 2003 \\
{[32]}\end{array}$ & 91 & 1 & 0.00549 & na \\
\hline $\begin{array}{l}\text { breast cancer, early } \\
\text { onset }\end{array}$ & $\begin{array}{l}\text { Musolino et al. } 2007 \\
\text { [33] }\end{array}$ & 66 & 3 & 0.02272 & na \\
\hline $\begin{array}{l}\text { breast cancer, early } \\
\text { onset }\end{array}$ & Juwle et al. 2012 [34] & 50 & 2 & 0.01 & na \\
\hline $\begin{array}{l}\text { breast cancer, early } \\
\text { onset }\end{array}$ & Juwle et al. 2012 [34] & 50 & 2 & 0.02 & na \\
\hline breast, ovarian cancer & Claes et al. 2003 [35] & 249 & 8 & 0.01606 & na \\
\hline breast, ovarian cancer & $\begin{array}{l}\text { Hadjisavvas et al. } \\
2003 \text { [36] }\end{array}$ & 26 & 1 & 0.01923 & na \\
\hline breast, ovarian cancer & $\begin{array}{c}\text { Giannini et al. } 2006 \\
\text { [37] }\end{array}$ & 73 & 1 & 0.00684 & na \\
\hline breast, ovarian cancer & $\begin{array}{l}\text { Simard et al. } 2007 \\
{[38]}\end{array}$ & 143 & 2 & 0.00699 & na \\
\hline breast, ovarian cancer & $\begin{array}{c}\text { Beristain et al. } 2007 \\
\text { [39] }\end{array}$ & 236 & 1 & 0.00211 & na \\
\hline breast, ovarian cancer & $\begin{array}{c}\text { Ratajska et al. } 2008 \\
\text { [18] }\end{array}$ & 64 & 0 & 0 & na \\
\hline breast, ovarian cancer & $\begin{array}{c}\text { Kuusisto et al. } 2011 \\
\text { [40] }\end{array}$ & 82 & 1 & 0.012 & $\begin{array}{c}\text { OR: } 0.41(0.05-3.24) ; \\
(p=0.702)\end{array}$ \\
\hline breast, ovarian cancer & $\begin{array}{l}\text { Cherbal et al. } 2012 \\
\text { [41] }\end{array}$ & 79 & 1 & 0.00632 & na \\
\hline breast, ovarian cancer & Jalkh et al. 2012 [42] & 72 & 1 & 0.00694 & na \\
\hline breast, ovarian cancer & $\begin{array}{l}\text { Dobričić et al. } 2013 \\
\text { [43] }\end{array}$ & 71 & 1 & 0.00704 & na \\
\hline breast, ovarian cancer & $\begin{array}{l}\text { Higgs et al. } \\
\text { 2015-cohort } 1 \text { [13] }\end{array}$ & 1850 & 23 & 0.00621 & \\
\hline breast, ovarian cancer & $\begin{array}{l}\text { Higgs et al. } \\
\text { 2015-cohort } 2 \text { [13] }\end{array}$ & 1576 & not reported & na & na \\
\hline
\end{tabular}


Table 4. Cont.

\begin{tabular}{|c|c|c|c|c|c|}
\hline Cancer Type & Reference & $\begin{array}{c}\text { Number of } \\
\text { Probands Screened }\end{array}$ & $\begin{array}{c}\text { Number of Patients } \\
\text { Carrying } B R C A 2 \\
\text { c.9976A }>\text { T Variant }\end{array}$ & Allelic Frequency & $\begin{array}{c}\text { Odds Ratio (OR) } \\
\text { (Patients vs. } \\
\text { Controls) } \\
\text { (Confidence } \\
\text { Intervals) }\end{array}$ \\
\hline breast, ovarian cancer & $\begin{array}{c}\text { Higgs et al. } \\
\text { 2015-cohort } 3 \text { [13] }\end{array}$ & 1395 & 43 & 0.01541 & \\
\hline ovarian cancer & $\begin{array}{c}\text { Mazoyer et al. } 1996 \\
\text { [11] }\end{array}$ & 361 & 7 & 0.00969 & na \\
\hline ovarian cancer & Hilton et al. 2002 [44] & 92 & 1 & 0.00543 & na \\
\hline ovarian cancer & Meeks et al. 2016 [15] & 14514 & 311 & 0.01071 & $\begin{array}{l}\text { OR: } 1.26(1.10-1.43) \\
\quad\left(p=3.84 \times 10^{-3}\right)\end{array}$ \\
\hline ovarian cancer & $\begin{array}{l}\text { Stafford et al. } 2017 \\
{[16]}\end{array}$ & 48 & $4^{*}$ & 0.00416 & OR: $4.95(p=0.01)$ \\
\hline male breast & $\begin{array}{l}\text { Haraldsson et al. } \\
1998[45]\end{array}$ & 34 & 1 & 0.01470 & na \\
\hline male breast & Ding et al. 2011 [46] & 115 & 2 & 0.00869 & na \\
\hline male breast & Evans et al. 2008 [47] & 64 & 1 & 0.00781 & na \\
\hline $\begin{array}{c}\text { familial pancreatic } \\
\text { cancer }\end{array}$ & Martin et al. 2005 [8] & 144 & 8 & 0.02777 & OR: $4.24(p<0.05)$ \\
\hline $\begin{array}{l}\text { sporadic pancreatic } \\
\text { cancer }\end{array}$ & $\begin{array}{c}\text { Obazee et al. } 2019 \\
\text { [48] }\end{array}$ & 2835 & 69 & 0.0123 & $\begin{array}{l}\text { OR: } 1.78(1.26-2.52) ; \\
\quad(p=0.00119)\end{array}$ \\
\hline lung cancer & Wang et al. 2014 [49] & 21435 & 298 & 0.01434 & $\begin{array}{l}\text { OR: } 1.83(\mathrm{OR})=2.47 \\
\quad\left(p=4.74 \times 10^{-20}\right)\end{array}$ \\
\hline lung cancer & Rudd et al. 2006 [50] & 1526 & 14 & 0.009 & $\begin{array}{l}\text { OR: } 1.72(0.15-2.57) ; \\
\quad(p=0.0075)\end{array}$ \\
\hline lung cancer & Rafnar et al. 2018 [51] & 4461 & na & na & $\begin{array}{l}\text { OR: } 1.54(1.23-1.91) ; \\
\quad(p=0.00012)\end{array}$ \\
\hline $\begin{array}{l}\text { incl: small cell lung } \\
\text { cancer }\end{array}$ & & 800 & na & na & OR: $2.06(1.35-3.16)$ \\
\hline $\begin{array}{l}\text { incl: squamous cell } \\
\text { lung carcinoma } \\
\text { (SQLC) }\end{array}$ & & 901 & na & na & $\begin{array}{c}\text { OR: } 1.71(1.10-2.67) ; \\
\quad(p=0.02)\end{array}$ \\
\hline $\begin{array}{l}\text { lung squamous cell } \\
\text { carcinoma }\end{array}$ & $\begin{array}{l}\text { Esai Selvan et al. } \\
2019 \text { [10] }\end{array}$ & 318 & na & na & $\begin{array}{l}\text { OR: } 3.0(1.4-6.4) \\
\quad(p=0.0053)\end{array}$ \\
\hline $\begin{array}{l}\text { esophageal } \\
\text { squamous cell } \\
\text { carcinoma }\end{array}$ & Akbari et al. 2008 [27] & 197 & 9 & 0.02284 & $\begin{array}{l}\text { OR: } 6.0(1.3-28) ; \\
\quad(p=0.01)\end{array}$ \\
\hline $\begin{array}{l}\text { UADT squamous cell } \\
\text { carcinoma }\end{array}$ & $\begin{array}{l}\text { Delahaye-Sourdeix } \\
\text { et al. } 2015 \text { [17] }\end{array}$ & 5942 & 149 & 0.01253 & $\begin{array}{l}\text { OR: } 2.53(1.89-3.38) ; \\
\quad\left(p=3 \times 10^{-10}\right)\end{array}$ \\
\hline bladder cancer & Ge et al. 2016 [52] & 3591 & 41 & 0.0096 & $\begin{array}{l}\text { OR: } 1.70(1.19-2.42) ; \\
\quad(p=0.0036)\end{array}$ \\
\hline renal cell carcinoma & Ge et al. 2016 [52] & 1322 & 13 & 0.0125 & $\begin{array}{c}\text { OR: } 1.60(0.91-2.82) ; \\
\quad(p=0.103)\end{array}$ \\
\hline prostate cancer & Ge et al. 2016 [52] & 1151 & 8 & 0.0076 & $\begin{array}{c}\text { OR: } 0.85(0.41-1.74) ; \\
(p=0.647)\end{array}$ \\
\hline $\begin{array}{l}\text { squamous cell } \\
\text { carcinoma of the skin }\end{array}$ & Rafnar et al. 2018 [51] & & & & OR: $1.69(1.26-2.26)$ \\
\hline melanoma & $\begin{array}{c}\text { Tuominen et al. } 2016 \\
\text { [53] }\end{array}$ & 452 & 12 & 0.01304 & $\begin{array}{c}\text { OR: } 2.80(1.04-7.58), \\
(p=0.035)\end{array}$ \\
\hline
\end{tabular}

SQLC: squamous cell lung carcinoma; UADT: upper aero-digestive tract.

Regarding the pathogenicity of the c.9976A $>$ T variant, the effect of linkage with the $B R C A 2$ c.6275_6276delTT variant has been previously raised. Data of nine studies were available regarding the status of the BRCA2 c.6275_6276delTT variant by analyzing 12,608 patients, including our results (Table 5). In one of the three cohorts reported by Higgs et al. 
(2015) and in the study by Meeks et al. (2016), increased carrier status of the deleterious variant $(25 / 1576$ and $233 / 306$, respectively) besides c.9976A $>\mathrm{T}$ was described $[13,15]$. Excluding these two studies among the remaining 12,232 cancer patients, c.9976A $>\mathrm{T}$ and c.6275_6276delTT co-carrier status was reported only in 50 cases $(0.4 \%)$.

Table 5. Studies analyzing linkage of c.9976A>T and c.6275_6276delTT.

\begin{tabular}{|c|c|c|c|c|c|c|}
\hline \multirow[t]{2}{*}{ Study } & \multirow[t]{2}{*}{ Note } & & \multirow[t]{2}{*}{$\begin{array}{c}\text { Number of Cases } \\
\text { Screened }\end{array}$} & \multirow{2}{*}{$\begin{array}{c}\text { Number of Cases } \\
\text { with } B R C A 2 \\
\text { c.9976A>T } \\
\text { Variant Alone }\end{array}$} & \multicolumn{2}{|c|}{$\begin{array}{l}\text { Number of Cases Carrying } \\
\text { BRCA2 c.9976A > T Variant WITH } \\
B R C A 2 \text { c.6275_6276delTT Variant }\end{array}$} \\
\hline & & & & & (\#) & $(\%)$ \\
\hline Current study & & breast, ovarian cancer & 2138 & 46 & 0 & 0 \\
\hline \multirow[t]{3}{*}{$\begin{array}{c}\text { Higgs et al. } 2015 \\
\text { [13] }\end{array}$} & $\begin{array}{l}\text { High-risk } \\
\text { breast/ovarian } \\
\text { cancer families, } \\
\text { Manchester } \\
\text { region of North } \\
\text { West England }\end{array}$ & breast, ovarian cancer & 1850 & 23 & 18 & 0.0097 \\
\hline & $\begin{array}{l}\text { Research study: } \\
\text { familial } \\
\text { breast/ovarian } \\
\text { cancer cases, } \\
\text { North West }\end{array}$ & breast, ovarian cancer & 1576 & not reported & 25 & 0.0159 \\
\hline & $\begin{array}{l}\text { Samples from } \\
\text { Liverpool (UK), } \\
\text { Irish Republic, } \\
\text { Finland and } \\
\text { Germany }\end{array}$ & breast, ovarian cancer & 1395 & 43 & 4 & 0.0029 \\
\hline $\begin{array}{l}\text { Mazoyer et al. } \\
1996[11]\end{array}$ & & breast cancer & 513 & 11 & 2 & 0.0039 \\
\hline $\begin{array}{l}\text { Mazoyer et al. } \\
1996[11]\end{array}$ & & ovarian cancer & 361 & 7 & 0 & 0 \\
\hline $\begin{array}{c}\text { Martin et al. } 2005 \\
{[8]}\end{array}$ & & $\begin{array}{l}\text { familial pancreatic } \\
\text { cancer }\end{array}$ & 144 & 8 & 0 & 0 \\
\hline $\begin{array}{l}\text { Akbari et al. } 2008 \\
\text { [27] }\end{array}$ & & $\begin{array}{c}\text { esophageal squamous } \\
\text { cell carcinoma }\end{array}$ & 197 & 9 & 0 & 0 \\
\hline $\begin{array}{l}\text { Wang et al. } 2014 \\
\text { [49] }\end{array}$ & $\begin{array}{c}\text { Meta-analysis of } 4 \\
\text { lung cancer } \\
\text { GWAS studies }\end{array}$ & lung cancer & 21,435 & 298 & $0 / 70$ & 0 \\
\hline \multirow{3}{*}{$\begin{array}{c}\text { Rafnar et al. } 2018 \\
\text { [51] }\end{array}$} & $\begin{array}{l}\text { Analysis of } 3 \\
\text { studies }\end{array}$ & lung cancer & 4461 & na & 0 & 0 \\
\hline & & $\begin{array}{l}\text { incl: small cell lung } \\
\text { cancer }\end{array}$ & 800 & na & 0 & 0 \\
\hline & & $\begin{array}{l}\text { incl: squamous cell } \\
\text { lung carcinoma } \\
\text { (SQLC) }\end{array}$ & 901 & na & 0 & 0 \\
\hline $\begin{array}{c}\text { Meeks et al. } 2016 \\
\text { [15] }\end{array}$ & & breast cancer & 41,081 & 852 & $233 / 306$ & 0.7614 \\
\hline $\begin{array}{l}\text { Haraldsson et al. } \\
1998 \text { [45] }\end{array}$ & & male breast cancer & 34 & 0 & 1 & 0.0294 \\
\hline
\end{tabular}

GWAS: genome wide association study.

\section{Discussion}

The clinical relevance of $B R C A 2 \mathrm{C}$-terminal stop codon variants remains controversial. The BRCA2 c.10095delinsGAATTATATCT variant located at the $3^{\prime}$ end of the gene is considered to be non-pathogenic based on the ENIGMA classification system. There are literature data regarding its allelic frequency and clinical relevance. Despite its low prevalence in control populations and its relatively higher frequency in breast/ovarian cancer patients, 
based on our and others' findings ( $\mathrm{LOH}$, allele imbalance, segregation and linkage data), this variant can be considered as clinically non-significant.

$B R C A 2$ c.9976A $>$ T, despite being a truncating variant, is usually classified as nonpathogenic based on case-control studies [11]. Indeed, in our study, the disease onset, tumor proliferation index or other pathological and clinical parameters did not differ in carriers compared to pathogenic $B R C A 2$ carriers or to $B R C A 1 / 2$ wild type patients. Additionally, we did not find an increased prevalence among carriers or in carrier families for HBOC. The MAF of c.9976A $>$ T is around 1\% among patients that also counts against its independent pathogenic role. The lack of genotype-phenotype segregation, lack of $\mathrm{LOH}$ and lack of allelic imbalance in patients are all in line with previous literature [11]. However, environmental factor-associated cancers (lung and skin carcinoma) were more frequent in families of the $B R C A 2$ c.9976A $>$ T carrier probands.

In previously published data, breast cancer risk was mildly elevated in BRCA2 c.9976A $>$ T carriers when compared to control populations in three reports $[9,14,15]$. Studies investigating only ovarian cancer patients, except that of Stafford et al. (2017) [16], showed similar ORs. In the study of Stafford et al., in all cases, the germline c.9976A $>$ T variant coexisted with other deleterious variants in other genes belonging to the $B R C A 2$ pathway. Among familial pancreatic, lung and upper aero-digestive tract (UADT) cancer patients, the c.9976A $>\mathrm{T}$ carrier status meant high odds (4.24, 3 and 2.53, respectively) for developing cancer $[8,10,17]$. In line with this, we observed an increased proportion of pancreatic cancer prevalence in families of double heterozygotes (c.9976A>T with pathogenic $B R C A 1$ variant), however, due to the limited number of cases, this observation should be validated in a larger sample cohort. Additionally, regarding pancreatic cancer, further analysis is subject to bias due to the secondary assessment of datasets. Others also suggested that the concomitant c.9976A $>$ T variant should be considered during genetic counseling for a potentially earlier age of HBOC cancer onset $[16,54,55]$. In the study of Akbari et al. (2008), c.9976A $>$ T carrier status was associated with a high OR of developing esophageal cancer $(6.0 ; 95 \%$ CI: 1.3-28; $p=0.01)$ [27]. Higgs et al. (2015) also reported multiple co-occurrences of the $B R C A 2$ c.9976A $>\mathrm{T}$ variant with the pathogenic $B R C A 2$ c.6275_6276delTT (p.(Leu2092ProfsTer7)) frameshift variant in breast and ovarian cancer patients [13]. The authors concluded that associations of increased cancer risk due to $B R C A 2$ c.9976A $>$ T represented a reporting bias and this was due to the variant being in linkage with $B R C A 2$ c.6275_6276delTT. However, in our patient cohort, neither investigated $C$-terminal stop codon variant was associated with any pathogenic $B R C A 2$ variant. Hence, we suggest that the linkage of the two $B R C A 2$ variants can be a founder phenomenon in the investigated cohort reported by Higgs et al. [13]. This is supported by other studies too [15], therefore, the reported variant associations may be a population specific-phenomenon representing a founder effect.

Although, based on our findings and previously published data, the BRCA2 c.9976A $>\mathrm{T}$ variant alone probably cannot be considered as a risk factor for breast and ovarian cancer, it seems to be associated with other cancer types. Genetic epidemiological evidence suggested that the $B R C A 2$ c. $9976 \mathrm{~A}>\mathrm{T}$ variant contributes to the risk of developing familial pancreatic cancer [8] and lung cancer [10,49,51]. Additionally, it was reported that the risk of developing lung cancer is approximately doubled for smokers compared to nonsmokers when carrying the c.9976A $>$ T variant $[17,49]$. Therefore, Wang et al. suggested that this finding may have implications for identifying high-risk ever-smoking subjects for lung cancer screening. Furthermore, it was reported [51] that the c.9976A $>$ T variant was associated with cancers that have strong environmental genotoxic risk factors. Based on functional studies, the authors proposed that the variant protein could probably retain the DNA repair capabilities important to hormone-responsive tissues but it might be less efficient in counteracting genotoxic stress [51]. In line with this, based on associations between this $B R C A 2$ variant and upper aero-digestive tract and lung cancer risk, PARP1 inhibitors were suggested as potential treatment strategies [17,49]. These findings have not been confirmed by functional studies investigating the role of the c.9976 A $>\mathrm{T}$ variant. Its 
damaging effects on the protein subcellular localization, cell viability, homology-directed repair (HDR) of double-strand breaks, centrosome amplification or sensitivity to DNA damaging agents $[56,57]$ were not observed. Moreover, it has been suggested that the protein, translated from the variant-carrier transcript, is defective in maintaining fork stability while being still proficient in HDR. Therefore, c.9976A $>$ T carriers may be insensitive to PARP inhibitors, which specifically exploits the defect of double-strand repair [7]. As a consequence, PARP-targeting therapy may not only be ineffective in these cases, but also induce further mutagenesis and genomic instability [7]. All these findings indicate that the clinical value of the use of PARP inhibitors in BRCA2 c.9976A>T carriers should be further investigated.

In summary, the clinical phenotypes associated with C-terminal BRCA2 variants are significantly different from those observed in families with highly penetrant $B R C A 2$ mutations $[58,59]$. For the expected pathogenic $B R C A 2$ mutation-associated cancer types (including breast, ovarian and prostate cancer), the C-terminal BRCA2 variants have not been found as risk factors [49,59]. However, these variants may be involved in the pathogenesis of pancreatic and environmental factor-associated cancers.

\section{Materials and Methods}

\subsection{Cases: Patients and Relatives}

We investigated 2491 independent patients (probands) with breast and/or ovarian cancer sent for germline BRCA1/2 genetic analysis to the Department of Molecular Genetics at the National Institute of Oncology, Hungary between 2014-2019. Only one variant carrier per family, the proband, was included in our analysis. Among them, the BRCA2 Cterminal stop codon variants (LRG_293t1:c.9976A>T and/or c.10095delinsGAATTATATCT) were identified in 49 cases (average age: $43.4 \pm 10.1$ years; 47 females, 2 males). Estrogen, progesterone, HER2 receptor status, Ki67 proliferation indices and histology were assessed as part of the routine diagnostics. All data were collected from the institutional medical information system. Details (patient characteristics and histology findings) are summarized in Table 1. The study was approved by the Scientific and Research Committee of the Medical Research Council of the Ministry of Health, Hungary (ETT-TUKEB 53720-4/2019/EÜIG). Fisher's exact test was used to examine the significance of the association (contingency) between phenotype and variant carrier status.

As a part of the Consortium of Investigators of Modifiers of BRCA1/2 (CIMBA) and the Breast Cancer Association Consortium (BCAC), standard phenotypic and epidemiological data collection was applied from 1998 during the study (http:/ / cimba.ccge.medschl.cam. ac.uk/ (accessed on 30 December 2020); http:/ / bcac.ccge.medschl.cam.ac.uk/bcacdata/ (accessed on 30 December 2020)). Details of data collection protocols have been used and reported previously $[60,61]$. Accordingly, for the analysis of phenotypic and pedigree data, standard questionnaires (one is patient/disease-centered and one for pedigree data) were sent out to all patients in advance. Based on the standard data, acquisition pedigrees were generated. During genetic counseling, data reliability was confirmed by reviewing all medical reports available by practicing clinical geneticists. In the family history analysis, three-generation pedigrees were investigated where only the presence of tumor types were considered (not the number of cases in each family).

\subsection{Nucleic Acid Extraction}

Germline variants were analyzed using total DNA extracted from peripheral blood using a Gentra Puregene Blood Kit (Cat No.: 158389, Qiagen, Hilden, Germany) following the manufacturer's instructions.

A GeneRead DNA FFPE Kit (Cat No.: 180134, Qiagen, Hilden, Germany) was applied to isolate genomic DNA from formalin-fixed paraffin-embedded (FFPE) tissues in an automated way using the QIAcube Instrument (Qiagen, Hilden, Germany).

RNA extraction was performed from total blood taken into Tempus ${ }^{\mathrm{TM}}$ Blood RNA Tubes (Thermo Fisher Scientific, Waltham, MA, USA) by a Tempus ${ }^{\mathrm{TM}}$ Spin RNA Isolation 
Kit. RNA quality and quantity were determined by a NanoDrop ${ }^{\circledR} 1000$ Spectrophotometer (NanoDrop Technologies, Thermo Fisher Scientific, Waltham, MA, USA).

4.3. Genetic Analysis (Sequence and Copy Number Analysis by Next Generation Sequencing (NGS) and Multiplex Ligation-Dependent Probe Amplification)

Genetic analyses were done as we previously reported [62]. Germline BRCA1/2 variant status was evaluated following library preparation using CE-IVD BRCA MASTR Plus Dx kit (Agilent, Santa Clara, CA, United States). Sequencing of the library was run on an Illumina MiSeq Instrument using MiSeq Reagent Kit v2 (500-cycles) (MS-102-2003, Illumina). Data analysis was done by MASTR Reporter software, a comprehensive CE-IVD marked (complies with the European In-Vitro Diagnostic Devices Directive) molecular solution for the identification of coding region variants in the BRCA1 and BRCA2 genes. Copy number analysis was performed by the Multiplex Ligation Dependent Probe Amplification (MLPA) method using P002 and P239 probe sets for BRCA1, and the P045 probe set for BRCA2 (MRC-Holland, the Netherlands).

Clinical significance of variants was evaluated and interpreted following AMCG/AMG recommendations [1], ENIGMA classification [2] and literature data mining.

The following transcripts were used for variant annotation. BRCA1: LRG_292t1 (NM_007294.3) and BRCA2: LRG_293t1 (NM_000059.3).

\subsection{Sanger Validation and LOH Analysis}

All germline pathogenic, likely pathogenic and variants of unknown significance (VUSs) were validated by traditional bidirectional Sanger sequencing on an independent blood sample. For loss-of-heterogeneity $(\mathrm{LOH})$ testing, DNA from tumor tissues was used for PCR amplification by a Qiagen Multiplex PCR Kit (Qiagen). PCR product was purified by ExoSAP-ITTM reagents (Thermo Fisher Scientific, Waltham, MA, United States), then purified amplicons were sequenced bidirectionally on an ABI3130 Genetic Analyzer (Applied Biosystems, Thermo Fisher Scientific, Waltham, MA, USA) using a BigDye ${ }^{\mathrm{TM}}$ Terminator v.1.1 kit (Thermo Fisher Scientific, Waltham, MA, USA).

\subsection{Transcript Allelic Imbalance}

Relative expression of the variant carrier and the normal allele was tested with Sanger sequencing. The ratio of electropherograms of the variant position on the cDNA template relative to the gDNA template was calculated. Briefly, cDNA was generated from 500 ng RNA using SuperScript ${ }^{\mathrm{TM}}$ IV Reverse Transcriptase (Thermo Fisher Scientific). cDNA primers were designed using Primer3Plus software (https//:primer3 plus.com): B2-C-e24_For-GATCCAGACTTTCAGCCATCTT and Rd_B2_Ex27.01_RevCGTCGTTTCAGTCTGAGATAATCT. Following PCR amplification and Sanger sequencing, data were visualized in Sequence Scanner software (Applied Biosystems, Thermo Fisher Scientific), and the peak ratio of the heterozygote position was given and compared to the peak ratio of the gDNA sequence of the same position for the same sample. The relative ratio was calculated and allelic imbalance was declared if the difference was $>50 \%$.

\subsection{Statistical Analysis}

For both proband characterization and family description, proportions and $95 \%$ confidence intervals by a modified Wald method were calculated using GraphPad QuickCalcs (https:/ / www.graphpad.com/quickcalcs/confInterval1/ (accessed on 30 December 2020)). For statistical analysis, $2 \times 2$ contingency tables were applied and $p$ values were calculated by Fisher's exact test. $p$ values were considered statistically significant at $<0.05$. Statistical power was calculated using the ClinCalc online algorithm (https://clincalc.com/stats / samplesize.aspx (accessed on 30 December 2020)). 


\section{Conclusions}

As a conclusion, our results suggest that among $B R C A 2 \mathrm{C}$-terminal stop codon variants, c.10095delinsGAATTATATCT is clinically non-significant. However, the c.9976A $>$ T variant may have different clinical significance compared to the $B R C A 2$ truncating variant before amino acid 3326. It may be considered as a genetic modifying factor in pancreas cancer when it co-occurs with pathogenic BRCA1 variants, although this observation should be validated in a larger sample cohort of double heterozygotes. Additionally, it seems to have an impact on the development of tumor types where environmental factors are significant as a genotoxic stress factor. Therefore, it is suggested to be a non-negligible variant, especially in the risk assessment of environmental cancers. The ACMG "pathogenic" classification is disease-specific. That is, a variant classified as (likely) benign with respect to HBOC still cannot be disregarded in conjunction with other, only loosely associated, diseases or with possible treatment options.

Additionally, our data, in line with a very recent review [63], suggest that collecting disease-specific clinical data regarding $C$-terminal $B R C A 2$ variants can assist in reducing the number of VUSs, which in turn may help in more precise treatment planning.

Supplementary Materials: The following are available online at https:/ / www.mdpi.com/2072-669 4/13/4/881/s1, Table S1: Family history of C-terminal stop codon carrier probands collected from three-generation pedigrees; Table S2: Supplementary Table 2. Fisher's exact $p$ values of comparison of tumor prevalence in families. Figure S1. Annotation of NM_000059.3 (BRCA2):c.10095delinsGAATTATATCT (p.Ser3366Asnfs*4) variant. A: c.10095delinsGAATTATATCT is annotated as two different variants (insertions) in gnomAD: NM_000059.4(BRCA2):c.10095_10096insT (p.Ser3366Ter) and NM_000059.3 (BRCA2):c.10094_10095insGAATTATAT (p.Ser3366_Glu3367insAsnTyrIle). B: Integrative Genomics Viewer (IGV) image of the "two misannotated variants". The cis allelic position of the c.10094_10095 insGAATTATAT and c.10095_10096insT variants are clearly visible on the IGV images of next generation sequencing data from gnomAD pages of both entries; they are always on the same reads of the pile-up IGV track and they were separated into their allelic primitives during variant calling.

Author Contributions: Conceptualization, H.B. and A.P.; methodology, J.P., H.B., A.B., T.P.; formal analysis, H.B., J.P., L.K.; investigation, J.P., H.B.; resources, A.P., E.O.; data curation, J.P.; writingoriginal draft preparation, H.B.; writing-review and editing, H.B., J.P., A.P., A.B.; supervision, A.P.; funding acquisition, A.P.; E.O. All authors have read and agreed to the published version of the manuscript.

Funding: The research was financed by a Hungarian Scientific Research Grant of the National Research, Development and Innovation Office (NKFI FK 135065) to Henriett Butz, National Bionics Program, medical bionics subtheme to Attila Patócs and Thematic Excellence Program (TKP2020NKA-26). Henriett Butz's work is supported by the ÚNKP-19-4 New National Excellence Program of the Ministry for Innovation and Technology.

Institutional Review Board Statement: The study was conducted according to the guidelines of the Declaration of Helsinki, and approved by the Scientific and Research Committee of the Medical Research Council of the Ministry of Health, Hungary (ETT-TUKEB 53720-4/2019/EÜIG).

Informed Consent Statement: Informed consent was obtained from all subjects involved in the study.

Data Availability Statement: All relevant data are included in the manuscript.

Conflicts of Interest: The authors declare no conflict of interest.

\section{References}

1. Richards, S.; Aziz, N.; Bale, S.; Bick, D.; Das, S.; Gastier-Foster, J.; Grody, W.W.; Hegde, M.; Lyon, E.; Spector, E.; et al. Standards and guidelines for the interpretation of sequence variants: A joint consensus recommendation of the American College of Medical Genetics and Genomics and the Association for Molecular Pathology. Genet. Med. 2015, 17, 405-423. [CrossRef]

2. Spurdle, A.B.; Healey, S.; Devereau, A.; Hogervorst, F.B.L.; Monteiro, A.N.A.; Nathanson, K.L.; Radice, P.; Stoppa-Lyonnet, D.; Tavtigian, S.; Wappenschmidt, B.; et al. ENIGMA-Evidence-based network for the interpretation of germline mutant alleles: An international initiative to evaluate risk and clinical significance associated with sequence variation in $B R C A 1$ and $B R C A 2$ genes. Hum. Mutat. 2011, 33, 2-7. [CrossRef] 
3. Fradet-Turcotte, A.; Sitz, J.; Grapton, D.; Orthwein, A. BRCA2 functions: From DNA repair to replication fork stabilization. Endocr. Relat. Cancer 2016, 23, T1-T17. [CrossRef]

4. Davies, O.R.; Pellegrini, L. Interaction with the BRCA2 C terminus protects RAD51-DNA filaments from disassembly by BRC repeats. Nat. Struct. Mol. Biol. 2007, 14, 475-483. [CrossRef] [PubMed]

5. Esashi, F.; Christ, N.; Gannon, J.; Liu, Y.; Hunt, T.L.; Jasin, M.; West, S.C. CDK-dependent phosphorylation of BRCA2 as a regulatory mechanism for recombinational repair. Nat. Cell Biol. 2005, 434, 598-604. [CrossRef]

6. Esashi, F.; Galkin, V.E.; Yu, X.; Egelman, E.H.; West, S.C. Stabilization of RAD51 nucleoprotein filaments by the C-terminal region of BRCA2. Nat. Struct. Mol. Biol. 2007, 14, 468-474. [CrossRef]

7. Schlacher, K.; Wu, H.; Jasin, M. A Distinct Replication Fork Protection Pathway Connects Fanconi Anemia Tumor Suppressors to RAD51-BRCA1/2. Cancer Cell 2012, 22, 106-116. [CrossRef]

8. Martin, S.T.; Matsubayashi, H.; Rogers, C.D.; Philips, J.; Couch, F.J.; Brune, K.; Yeo, C.J.; Kern, S.E.; Hruban, R.H.; Goggins, M. Increased prevalence of the BRCA2 polymorphic stop codon K3326X among individuals with familial pancreatic cancer. Oncogene 2005, 24, 3652-3656. [CrossRef]

9. Thompson, E.R.; Gorringe, K.L.; Rowley, S.M.; Li, N.; McInerny, S.; Wong-Brown, M.W.; Devereux, L.; Li, J.; Trainer, A.H.; Mitchell, G.; et al. Reevaluation of the BRCA2 truncating allele c.9976A > T (p.Lys3326Ter) in a familial breast cancer context. Sci. Rep. 2015, 5, 14800. [CrossRef]

10. Selvan, M.E.; Klein, R.J.; Gümüş, Z.H. Rare, Pathogenic Germline Variants in Fanconi Anemia Genes Increase Risk for Squamous Lung Cancer. Clin. Cancer Res. 2019, 25, 1517-1525. [CrossRef] [PubMed]

11. Mazoyer, S.; Dunning, A.M.; Serova, O.; Dearden, J.; Puget, N.; Healey, C.S.; Gayther, S.A.; Mangion, J.; Stratton, M.R.; Lynch, H.T.; et al. A polymorphic stop codon in BRCA2. Nat. Genet. 1996, 14, 253-254. [CrossRef]

12. Johnson, N.; Fletcher, O.; Palles, C.; Rudd, M.; Webb, E.; Sellick, G.; Silva, I.D.S.; McCormack, V.; Gibson, L.; Fraser, A.; et al. Counting potentially functional variants in BRCA1, BRCA2 and ATM predicts breast cancer susceptibility. Hum. Mol. Genet. 2007, 16, 1051-1057. [CrossRef]

13. Higgs, J.E.; Harkness, E.F.; Bowers, N.L.; Howard, E.; Wallace, A.J.; Lalloo, F.; Newman, W.G.; Evans, D.G. The BRCA2 Polymorphic Stop Codon: Stuff or Nonsense? J. Med. Genet. 2015, 52, 642-645. [CrossRef] [PubMed]

14. Michailidou, K.; Hall, P.; Gonzalez-Neira, A.; Ghoussaini, M.; Dennis, J.; Milne, R.L.; Schmidt, M.K.; Chang-Claude, J.; Bojesen, S.E.; Bolla, M.K.; et al. Large-scale genotyping identifies 41 new loci associated with breast cancer risk. Nat. Genet. 2013, 45, 353-361. [CrossRef]

15. Meeks, H.D.; Song, H.; Michailidou, K.; Bolla, M.K.; Dennis, J.; Wang, Q.; Barrowdale, D.; Frost, D.; McGuffog, L.; Ellis, S.; et al. BRCA2 Polymorphic Stop Codon K3326X and the Risk of Breast, Prostate, and Ovarian Cancers. J. Natl. Cancer Inst. 2015, 108. [CrossRef]

16. Stafford, J.L.; Dyson, G.; Levin, N.K.; Chaudhry, S.; Rosati, R.; Kalpage, H.; Wernette, C.; Petrucelli, N.; Simon, M.S.; Tainsky, M.A. Reanalysis of $B R C A 1 / 2$ negative high risk ovarian cancer patients reveals novel germline risk loci and insights into missing heritability. PLOS ONE 2017, 12, e0178450. [CrossRef]

17. Delahaye-Sourdeix, M.; Anantharaman, D.; Timofeeva, M.N.; Gaborieau, V.; Chabrier, A.; Vallée, M.P.; Lagiou, P.; Holcátová, I.; Richiardi, L.; Kjaerheim, K.; et al. A Rare Truncating BRCA2 Variant and Genetic Susceptibility to Upper Aerodigestive Tract Cancer. J. Natl. Cancer Inst. 2015, 107. [CrossRef]

18. Ratajska, M.; Brozek, I.; Senkus-Konefka, E.; Jassem, J.; Stepnowska, M.; Palomba, G.; Pisano, M.; Casula, M.; Palmieri, G.; Borg, A.; et al. BRCA1 and BRCA2 point mutations and large rearrangements in breast and ovarian cancer families in Northern Poland. Oncol. Rep. 2008, 19, 263-268. [CrossRef] [PubMed]

19. Machackova, E.; Foretova, L.; Lukesova, M.; Vasickova, P.; Navratilova, M.; Coene, I.; Pavlu, H.; Kosinova, V.; Kuklova, J.; Claes, K. Spectrum and characterisation of $B R C A 1$ and $B R C A 2$ deleterious mutations in high-risk Czech patients with breast and/or ovarian cancer. BMC Cancer 2008, 8, 140. [CrossRef] [PubMed]

20. German Consortium for Hereditary Breast and Ovarian Cancer Comprehensive analysis of 989 patients with breast or ovarian cancer provides BRCA1 and BRCA2 mutation profiles and frequencies for the German population. Int. J. Cancer 2002, 97, 472-480. [CrossRef]

21. Cvok, M.L.; Sabol, M.; Musani, V.; Ozretić, P.; Levanat, S. New sequence variants in BRCA1 and BRCA2 genes detected by high-resolution melting analysis in an elderly healthy female population in Croatia. Clin. Chem. Lab. Med. 2008, 46, 1376-1383. [CrossRef] [PubMed]

22. Hahn, S.A.; Greenhalf, B.; Ellis, I.; Sina-Frey, M.; Rieder, H.; Korte, B.; Gerdes, B.; Kress, R.; Ziegler, A.; Raeburn, J.A.; et al. BRCA2 Germline Mutations in Familial Pancreatic Carcinoma. J. Natl. Cancer Inst. 2003, 95, 214-221. [CrossRef]

23. Koczkowska, M.; Zuk, M.; Gorczynski, A.; Ratajska, M.; Lewandowska, M.; Biernat, W.; Limon, J.; Wasag, B. Detection of somatic BRCA 1/2 mutations in ovarian cancer-Next-generation sequencing analysis of 100 cases. Cancer Med. 2016, 5, 1640-1646. [CrossRef]

24. Caminsky, N.G.; Mucaki, E.J.; Perri, A.M.; Lu, R.; Knoll, J.H.M.; Rogan, P.K. Prioritizing Variants in Complete Hereditary Breast and Ovarian Cancer Genes in Patients Lacking Known BRCA Mutations. Hum. Mutat. 2016, 37, 640-652. [CrossRef] [PubMed]

25. Thomassen, M.; Hansen, T.V.O.; Borg, A.; Lianee, H.T.; Wikman, F.; Pedersen, I.S.; Bisgaard, M.L.; Nielsen, F.C.; Kruse, T.A.; Gerdes, A.-M. BRCA1 and BRCA2 mutations in Danish families with hereditary breast and/or ovarian cancer. Acta Oncol. 2008, 47, 772-777. [CrossRef] [PubMed] 
26. Meisel, C.; Sadowski, C.E.; Kohlstedt, D.; Keller, K.; Stäritz, F.; Grübling, N.; Becker, K.; Mackenroth, L.; Rump, A.; Schröck, E.; et al. Spectrum of genetic variants of BRCA1 and BRCA2 in a German single center study. Arch. Gynecol. Obstet. 2017, 295, 1227-1238. [CrossRef] [PubMed]

27. Akbari, M.R.; Malekzadeh, R.; Nasrollahzadeh, D.; Amanian, D.; Islami, F.; Li, S.; Zandvakili, I.; Shakeri, R.; Sotoudeh, M.; Aghcheli, K.; et al. Germline BRCA2 mutations and the risk of esophageal squamous cell carcinoma. Oncogene 2007, 27, 1290-1296. [CrossRef]

28. Borg, Å.; Haile, R.W.; Malone, K.E.; Capanu, M.; Diep, A.; Törngren, T.; Teraoka, S.; Begg, C.B.; Thomas, D.C.; Concannon, P.; et al. Characterization of $B R C A 1$ and $B R C A 2$ deleterious mutations and variants of unknown clinical significance in unilateral and bilateral breast cancer: The WECARE study. Hum. Mutat. 2010, 31, E1200-E1240. [CrossRef]

29. Krainer, M.; Silva-Arrieta, S.; Fitzgerald, M.G.; Shimada, A.; Ishioka, C.; Kanamaru, R.; Macdonald, D.J.; Unsal, H.; Finkelstein, D.M.; Bowcock, A.; et al. Differential Contributions of BRCA1 and BRCA2 to Early-Onset Breast Cancer. N. Engl. J. Med. 1997, 336, 1416-1422. [CrossRef]

30. Malone, K.E.; Daling, J.R.; Neal, C.; Suter, N.M.; O’Brien, C.; Cushing-Haugen, K.; Jonasdottir, T.J.; Thompson, J.D.; Ostrander, E.A. Frequency of BRCA1/BRCA2 mutations in a population-based sample of young breast carcinoma cases. Cancer 2000, 88, 1393-1402. [CrossRef]

31. Bergthorsson, J.; Ejlertsen, B.; Olsen, J.; Borg, Å.; Nielsen, K.; Barkardottir, R.; Klausen, S.; Mouridsen, H.; Winther, K.; Fenger, K.; et al. BRCA1 and BRCA2 mutation status and cancer family history of Danish women affected with multifocal or bilateral breast cancer at a young age. J. Med. Genet. 2001, 38, 361-368. [CrossRef]

32. Hamann, U.; Liu, X.; Bungardt, N.; Ulmer, H.U.; Bastert, G.; Sinn, H.-P. Similar contributions of BRCA1 and BRCA2 germline mutations to early-onset breast cancer in Germany. Eur. J. Hum. Genet. 2003, 11, 464-467. [CrossRef] [PubMed]

33. Musolino, A.; Bella, M.A.; Bortesi, B.; Michiara, M.; Naldi, N.; Zanelli, P.; Capelletti, M.; Pezzuolo, D.; Camisa, R.; Savi, M.; et al. BRCA mutations, molecular markers, and clinical variables in early-onset breast cancer: A population-based study. Breast 2007, 16, 280-292. [CrossRef]

34. Juwle, A.; Saranath, D. BRCA1/BRCA2 gene mutations/SNPs and BRCA1 haplotypes in early-onset breast cancer patients of Indian ethnicity. Med. Oncol. 2012, 29, 3272-3281. [CrossRef]

35. Claes, K.; Poppe, B.; Machackova, E.; Coene, I.; Foretova, L.; de Paepe, A.; Messiaen, L. Differentiating pathogenic mutations from polymorphic alterations in the splice sites of BRCA1 and BRCA2. Genes Chromosom. Cancer 2003, 37, 314-320. [CrossRef] [PubMed]

36. Hadjisavvas, A.; Charalambous, E.; Adamou, A.; Christodoulou, C.G.; Kyriacou, K. BRCA2 germline mutations in Cypriot patients with familial breast/ovarian cancer. Hum. Mutat. 2003, 21, 171. [CrossRef]

37. Giannini, G.; Capalbo, C.; Ristori, E.; Ricevuto, E.; Sidoni, T.; Buffone, A.; Cortesi, E.; Marchetti, P.; Scambia, G.; Tomao, S.; et al. Novel BRCA1 and BRCA2 germline mutations and assessment of mutation spectrum and prevalence in Italian breast and/or ovarian cancer families. Breast Cancer Res. Treat. 2006, 100, 83-91. [CrossRef]

38. Simard, J.-C.; Dumont, M.; Moisan, A.-M.; Gaborieau, V.; Vézina, H.; Durocher, F.; Chiquette, J.; Plante, M.; Avard, D.; Bessette, P.; et al. Evaluation of $B R C A 1$ and $B R C A 2$ mutation prevalence, risk prediction models and a multistep testing approach in French-Canadian families with high risk of breast and ovarian cancer. J. Med. Genet. 2006, 44, 107-121. [CrossRef]

39. Beristain, E.; Martínez-Bouzas, C.; Guerra, I.; Viguera, N.; Moreno, J.; Ibáñez, E.; Diez, J.; Rodríguez, F.; Mallabiabarrena, G.; Luján, S.; et al. Differences in the frequency and distribution of BRCA1 and BRCA2 mutations in breast/ovarian cancer cases from the Basque country with respect to the Spanish population: Implications for genetic counselling. Breast Cancer Res. Treat. 2007, 106, 255-262. [CrossRef]

40. Kuusisto, K.M.; Bebel, A.; Vihinen, M.; Schleutker, J.; Sallinen, S.-L. Screening for BRCA1, BRCA2, CHEK2, PALB2, BRIP1, RAD50, and $C D H 1$ mutations in high-risk Finnish BRCA1/2-founder mutation-negative breast and/or ovarian cancer individuals. Breast Cancer Res. 2011, 13, R20. [CrossRef]

41. Cherbal, F.; Salhi, N.; Bakour, R.; Adane, S.; Boualga, K.; Maillet, P. BRCA1 and BRCA2 Unclassified Variants and Missense Polymorphisms in Algerian Breast/Ovarian Cancer Families. Dis. Markers 2012, 32, 343-353. [CrossRef] [PubMed]

42. Jalkh, N.; Nassar-Slaba, J.; Chouery, E.; Salem, N.; Uhrchammer, N.; Golmard, L.; Stoppa-Lyonnet, D.; Bignon, Y.-J.; Mégarbané, A. Prevalance of BRCA1 and BRCA2 mutations in familial breast cancer patients in Lebanon. Hered. Cancer Clin. Pr. 2012, 10, 7. [CrossRef]

43. Dobričić, J.; Krivokuća, A.; Brotto, K.; Mališić, E.; Radulović, S.; Branković-Magić, M. Serbian high-risk families: Extensive results on BRCA mutation spectra and frequency. J. Hum. Genet. 2013, 58, 501-507. [CrossRef]

44. Hilton, J.L.; Geisler, J.P.; Rathe, J.A.; Hattermann-Zogg, M.A.; Deyoung, B.; Buller, R.E. Inactivation of BRCA1 and BRCA2 in Ovarian Cancer. J. Natl. Cancer Inst. 2002, 94, 1396-1406. [CrossRef]

45. Haraldsson, K.; Loman, N.; Zhang, Q.X.; Johannsson, O.; Olsson, H.; Borg, A. BRCA2 germ-line mutations are frequent in male breast cancer patients without a family history of the disease. Cancer Res. 1998, 58, 1367-1371. [PubMed]

46. Ding, Y.C.; Steele, L.; Kuan, C.-J.; Greilac, S.; Neuhausen, S.L. Mutations in BRCA2 and PALB2 in male breast cancer cases from the United States. Breast Cancer Res. Treat. 2010, 126, 771-778. [CrossRef] [PubMed]

47. Evans, D.G.R.; Bulman, M.; Young, K.; Howard, E.; Bayliss, S.; Wallace, A.; Lalloo, F. BRCA1/2 mutation analysis in male breast cancer families from North West England. Fam. Cancer 2007, 7, 113-117. [CrossRef] 
48. Obazee, O.; Archibugi, L.; Andriulli, A.; Soucek, P.; Małecka-Panas, E.; Ivanauskas, A.; Johnson, T.; Gazouli, M.; Pausch, T.; Lawlor, R.T.; et al. Germline BRCA2 K3326X and CHEK2 I157T mutations increase risk for sporadic pancreatic ductal adenocarcinoma. Int. J. Cancer 2019, 145, 686-693. [CrossRef]

49. Wang, Y.; McKay, J.D.; Rafnar, T.; Wang, Z.; Timofeeva, M.N.; Broderick, P.; Zong, X.; Laplana, M.; Wei, Y.; Han, Y.; et al. Rare variants of large effect in BRCA2 and CHEK2 affect risk of lung cancer. Nat. Genet. 2014, 46, 736-741. [CrossRef]

50. Rudd, M.F.; Webb, E.L.; Matakidou, A.; Sellick, G.S.; Williams, R.D.; Bridle, H.; Eisen, T.; Houlston, R.S. Variants in the GH-IGF axis confer susceptibilityto lung cancer. Genome Res. 2006, 16, 693-701. [CrossRef]

51. Rafnar, T.; Sigurjonsdottir, G.R.; Stacey, S.N.; Halldorsson, G.; Sulem, P.; Pardo, L.M.; Helgason, H.; Sigurdsson, S.T.; Gudjonsson, T.; Tryggvadottir, L.; et al. Association of BRCA2 K3326* With Small Cell Lung Cancer and Squamous Cell Cancer of the Skin. J. Natl. Cancer Inst. 2018, 110, 967-974. [CrossRef]

52. Ge, Y.; Wang, Y.; Shao, W.; Jin, J.; Du, M.; Ma, G.; Chu, H.; Wang, M.; Zhang, Z. Rare variants in BRCA2 and CHEK2 are associated with the risk of urinary tract cancers. Sci. Rep. 2016, 6, 33542. [CrossRef]

53. Tuominen, R.; Engström, P.G.; Helgadottir, H.; Eriksson, H.; Unneberg, P.; Kjellqvist, S.; Yang, M.; Lindén, D.; Edsgärd, D.; Hansson, J.; et al. The role of germline alterations in the DNA damage response genes BRIP1 and BRCA2 in melanoma susceptibility. Genes Chromosom. Cancer 2016, 55, 601-611. [CrossRef]

54. Palmirotta, R.; Lovero, D.; Stucci, L.S.; Silvestris, E.; Quaresmini, D.; Cardascia, A.; Silvestris, F. Double Heterozygosity for BRCA1 Pathogenic Variant and BRCA2 Polymorphic Stop Codon K3326X: A Case Report in a Southern Italian Family. Int. J. Mol. Sci. 2018, 19, 285. [CrossRef]

55. Heidemann, S.; Fischer, C.; Engel, C.; Fischer, B.; Harder, L.; Schlegelberger, B.; Niederacher, D.; Goecke, T.O.; Doelken, S.C.; Dikow, N.; et al. Double heterozygosity for mutations in BRCA1 and BRCA2 in German breast cancer patients: Implications on test strategies and clinical management. Breast Cancer Res. Treat. 2012, 134, 1229-1239. [CrossRef] [PubMed]

56. Wu, K.; Hinson, S.R.; Ohashi, A.; Farrugia, D.; Wendt, P.; Tavtigian, S.V.; Deffenbaugh, A.; Goldgar, D.; Couch, F.J. Functional evaluation and cancer risk assessment of BRCA2 unclassified variants. Cancer Res. 2005, 65, 417-426. [PubMed]

57. Kuznetsov, S.G.; Liu, P.; Sharan, S.K. Mouse embryonic stem cell-based functional assay to evaluate mutations in BRCA2. Nat. Med. 2008, 14, 875-881. [CrossRef]

58. Easton, D.F. Breast Cancer Linkage Consortium Cancer Risks in BRCA2 Mutation Carriers. J. Natl. Cancer Inst. 1999, 91, 1310-1316. [CrossRef]

59. Van Asperen, C.J.; Brohet, R.M.; Meijers-Heijboer, E.J.; Hoogerbrugge, N.; Verhoef, S.; Vasen, H.F.A.; Ausems, M.G.E.M.; Menko, F.H.; Garcia, E.B.G.; Klijn, J.G.M.; et al. Cancer risks in BRCA2 families: Estimates for sites other than breast and ovary. J. Med. Genet. 2005, 42, 711-719. [CrossRef] [PubMed]

60. Antoniou, A.C.; Sinilnikova, O.M.; Simard, J.; Léoné, M.; Dumont, M.; Neuhausen, S.L.; Struewing, J.P.; Stoppa-Lyonnet, D.; Barjhoux, L.; Hughes, D.J.; et al. RAD51 135G $\rightarrow$ C Modifies Breast Cancer Risk among BRCA2 Mutation Carriers: Results from a Combined Analysis of 19 Studies. Am. J. Hum. Genet. 2007, 81, 1186-1200. [CrossRef] [PubMed]

61. Rebbeck, T.R.; Friebel, T.M.; Friedman, E.; Hamann, U.; Huo, D.; Kwong, A.; Olah, E.; Olopade, O.I.; Solano, A.R.; Teo, S.-H.; et al. Mutational spectrum in a worldwide study of 29,700 families with BRCA 1or BRCA2 mutations. Hum. Mutat. 2018, 39, 593-620. [CrossRef] [PubMed]

62. Bozsik, A.; Pócza, T.; Papp, J.; Vaszkó, T.; Butz, H.; Patócs, A.; Oláh, E. Complex Characterization of Germline Large Genomic Rearrangements of the BRCA1 and BRCA2 Genes in High-Risk Breast Cancer Patients-Novel Variants from a Large National Center. Int. J. Mol. Sci. 2020, 21, 4650. [CrossRef] [PubMed]

63. Baughan, S.; Tainsky, M. K3326X and Other C-Terminal BRCA2 Variants Implicated in Hereditary Cancer Syndromes: A Review. Cancers 2021, 13, 447. [CrossRef] [PubMed] 